\title{
BRUTUS-LIKE proteins moderate the transcriptional response to iron deficiency in roots
}

Authors: Jorge Rodríguez-Celma ${ }^{1,2}$, Robert T. Green ${ }^{1}$, James M. Connorton ${ }^{1,2}$, Inga Kruse ${ }^{1}$, Yan Cui ${ }^{3}$, Hong Qing Ling ${ }^{3}$, Janneke Balk*1,2

1 John Innes Centre, Department of Biological Chemistry, NR4 7UH, United Kingdom

${ }^{2}$ University of East Anglia, School of Biological Sciences, NR4 7TJ, United Kingdom

${ }^{3}$ Institute of Genetics and Developmental Biology, Chinese Academy of Sciences, Beijing, China *corresponding author: Janneke Balk, janneke.balk@jic.ac.uk

Short title: BRUTUS-LIKE proteins target FIT for degradation

The author responsible for distribution of materials integral to the findings presented in this article in accordance with the policy described in the Instructions for Authors (www.plantcell.org) is: Janneke Balk (janneke.balk@jic.ac.uk). 


\section{ABSTRACT}

Iron is an essential micronutrient but in excess is toxic inside cells. Under iron deficiency, the expression of iron uptake genes is increased, but it is not known how the transcriptional response is controlled to avoid uptake of too much iron. The hemerythrin E3 ligases BRUTUS (BTS) and BTS-LIKE (BTSL) have previously been identified as negative regulators of the iron deficiency response. Our phylogenetic analysis indicated that BTSL proteins are present in dicotyledonous plants only and form a separate clade from BTS homologs. BTSL1 and BTSL2 in Arabidopsis thaliana are in a network with nearly all iron uptake genes, whereas BTS is in a shoot-specific network. BTSL1 and BTSL2 are expressed predominantly in the root epidermis and cortex, separate from BTS in the root stele, shoot and embryos. Mutant analysis identified BTSL2 as the dominant paralog of the otherwise redundant BTSL genes. The bts/ double mutant had increased protein levels of FIT, the FER-like Iron deficiency-induced Transcription factor, and failed to switch off the transcriptional response upon iron resupply, leading to dramatic iron accumulation in roots and shoots. Protein interaction between the $\mathrm{C}$-terminus of BTSL proteins and FIT indicate that FIT is a direct target for degradation. Taken together, our studies show that BTSL1 and BTSL2 control iron uptake in the epidermis and cortex, upstream of BTS in the vasculature and leaves. 


\section{INTRODUCTION}

Iron (Fe) is the fourth most abundant element in the Earth's crust, but its bioavailability is greatly limited by the insolubility of Fe hydroxides. Plants have developed highly efficient $\mathrm{Fe}$ uptake systems, classically divided in Strategy I or reductive strategy, present in dicotyledonous plants, and Strategy II or chelating strategy in grasses (Römheld and Marschner, 1986; Tsai and Schmidt, 2017). The reductive strategy in plants such as Arabidopsis involves the activity of a Ferric Reductive Oxidase, $\mathrm{FRO} 2$, to reduce $\mathrm{Fe}^{3+}$ to $\mathrm{Fe}^{2+}$ which is then taken up by the Iron-Regulated Transporter IRT1. A key regulator of Fe uptake in Strategy I plants is the basic Helix-Loop-Helix (bHLH) transcription factor FIT (FER-like Iron deficiency-induced Transcription factor; Bauer et al., 2007). FIT forms heterodimers with one of four bHLH proteins from subgroup lb, namely bHLH38, bHLH39, bHLH100 and bHLH101. Mutant studies combined with transcriptomics have identified more than 400 genes that are controlled by FIT (Colangelo and Guerinot, 2004; Mai et al., 2016). These targets include FRO2 and IRT1 for which direct promoter binding by the FIT-bHLH Ib dimer has been shown (Yuan et al., 2008; Wang et al., 2013).

How the Fe status of the plant is communicated to the transcriptional regulators remains unknown. Recent studies have discovered a small family of hemerythrin E3 ligases in plants, including BRUTUS (BTS) in Arabidopsis (Long et al., 2010; Selote et al., 2015) and Hemerythrin motif-containing RING- and Zn-finger proteins (HRZ1, HRZ2) in rice (Kobayashi et al., 2013), that act as negative regulators of the Fe deficiency response. The expression of $H R Z 1, H R Z 2$ and BTS is induced $\sim 10$-fold under Fe deficiency. Mutant lines of BTS or HRZ showed increased tolerance to Fe deficiency and accumulation of $\mathrm{Fe}$ in leaves and seeds. BTS / HRZ proteins contain three putative Fe-binding hemerythrin domains in the $\mathrm{N}$-terminus, and a CHY/RING-type Zn finger domain with ubiquitin E3 ligase activity in the C-terminus (Kobayashi et al., 2013; Selote et al., 2015). This domain architecture bears similarity to mammalian FBXL5 (F-box/LRR-repeat protein 5), which targets the Iron Regulatory Protein 2 (IRP2) for degradation (Vashisht et al., 2009; Salahudeen et al., 2009). The activity of FBXL5 depends on $\mathrm{Fe}$ binding to its N-terminal hemerythrin domain and on oxygen levels (Salahudeen et al., 2009). Plants do not have a functional homolog of IRP2, however, BTS was shown to interact with, and affect the stability of bHLH105 (ILR3) and bHLH115, two Feregulated transcription factors (Selote et al., 2015). A heterodimer of ILR3 and bHLH104 regulates the expression of $b H L H 38$ / $39 / 100 / 101$, encoding the main protein partners of FIT (Zhang et al., 2016).

BTS was first identified as EMBRYO DEFECTIVE 2454 (EMB2454) in a screen for embryo lethal mutants (McElver et al., 2001; Tzafrir et al., 2004), and later re-named and described 
as a negative regulator of Fe homeostasis (Long et al., 2010). Since bts loss-of-function mutants are lethal, studies have focused on viable alleles with T-DNA insertion in the promoter/UTRs or point mutations (Selote et al., 2015; Hindt et al., 2017). These bts mutant plants showed an enhanced Fe deficiency response, including increased root length, soil acidification and higher Fe reductase activities, and they are more tolerant to Fe limiting conditions. Promoter-GUS lines showed that BTS is expressed in leaves and embryos independent of the Fe status of the plant, and its expression is induced in the stele of the root under Fe deficiency (Selote et al., 2015). BTS is not the only hemerythrin-E3 ligase in Arabidopsis, as previously noticed by Kobayashi and colleagues (2013). Two homologous genes are found in the Arabidopsis genome, BRUTUS-Like1 (AT1G74770) and BRUTUSLike2 (AT1G18910). Double bts/1 btsl2 mutants have recently been reported to be more tolerant to Fe deficiency and to accumulate more Fe (Hindt et al., 2017). A triple mutant of $b t s / 1, b t s / 2$ and a viable bts allele had an enhanced phenotype, and the authors concluded that the BTSL genes act as redundant negative regulators of Fe homeostasis together with BTS (Hindt et al., 2017).

Here we show that the function of the BTSL genes differs from BTS. BTSL1 and BTSL2 are expressed predominantly in the root epidermis and cortex and are part of a root-specific transcriptional network of genes involved in Fe uptake. In contrast, BTS is co-expressed with only a small number of shoot-specific Fe-regulated genes. We found that BTSL1/2 function to control the levels of FIT protein and prevent excess Fe accumulation via the FRO2 / IRT1 uptake pathway. 


\section{RESULTS}

\section{BTSL1 and BTSL2 are co-expressed with Fe-responsive genes in the roots}

Co-expression analysis is a powerful tool for functional characterization because co-regulated genes are likely to function in the same biochemical pathway or biological process (Stuart et al., 2003). Previously we surveyed the transcriptional response to Fe deficiency in Arabidopsis and in Medicago truncatula (Rodríguez-Celma et al., 2013a). We considered the overlap between the two data sets working under the assumption that Fe homeostasis regulation should be conserved between plant species. We found the orthologous pair AT1G18910Medtr7g037040 up-regulated under Fe deficiency with the core genes of the Fe regulon. This gene has recently been annotated as BTSL2 (Hindt et al., 2017). As an independent and unbiased approach, we used publicly available microarray data to build co-expression networks around BTSL2 and the two homologous genes BTSL1 and BTS. First, we found that BTSL1 and BTSL2 are co-regulated with each other but not with BTS. Second, we noticed that a large network could be built around BTSL1 and BTSL2 with root-specific data sets, but no co-regulated 'root' partners could be found for BTS (Supplementary Figure S1 and Supplementary Table S1). Correlations with BTS were only found when using shoot-specific data sets. Because BTS, BTSL1 and BTSL2 have been linked to the Fe deficiency response, the networks were trimmed to only those genes present in the so-called ferrome (Schmidt and Buckhout, 2011) (Figure 1A, B, Supplementary Table S2). We found that BTSL1 and BTSL2 are co-regulated with genes involved in Fe uptake, such as the Fe transporter IRT1 and genes for the biosynthesis and export of coumarin-derived Fe chelators (PDR9, 4CL2 and F6'H1) (Figure 1A). Also co-expressed with BTSL1 and BTSL2 are the transcription factor FIT and its partner $b H L H 39$, central regulators in the Fe-deficiency response (Figure 1B). Basically, the entire root Fe uptake regulon is found, except for $b H L H 38$ and $F R O 2$, but these genes are not present on the micro-array chip (ATH1) that is most commonly used in the public database. The BTS network includes the transcription factor POPEYE (PYE), as previously documented (Long et al., 2010), and the ferric reductase FRO3. Also found is AT5G05250, a gene of unknown function which is highly induced during Fe deficiency (Rodriguez-Celma et al., 2013b).

Investigation of the expression of BTS, BTSL1 and BTSL2 in RNAseq data sets of the Fe deficiency response (Rodríguez-Celma et al., 2013b) confirmed that BTSL1 and BTSL2 were expressed mainly in the roots and strongly induced under Fe deficiency (Figure 1C). BTS is therefore the dominant family member expressed in the shoots, in accordance with the calculated networks. 

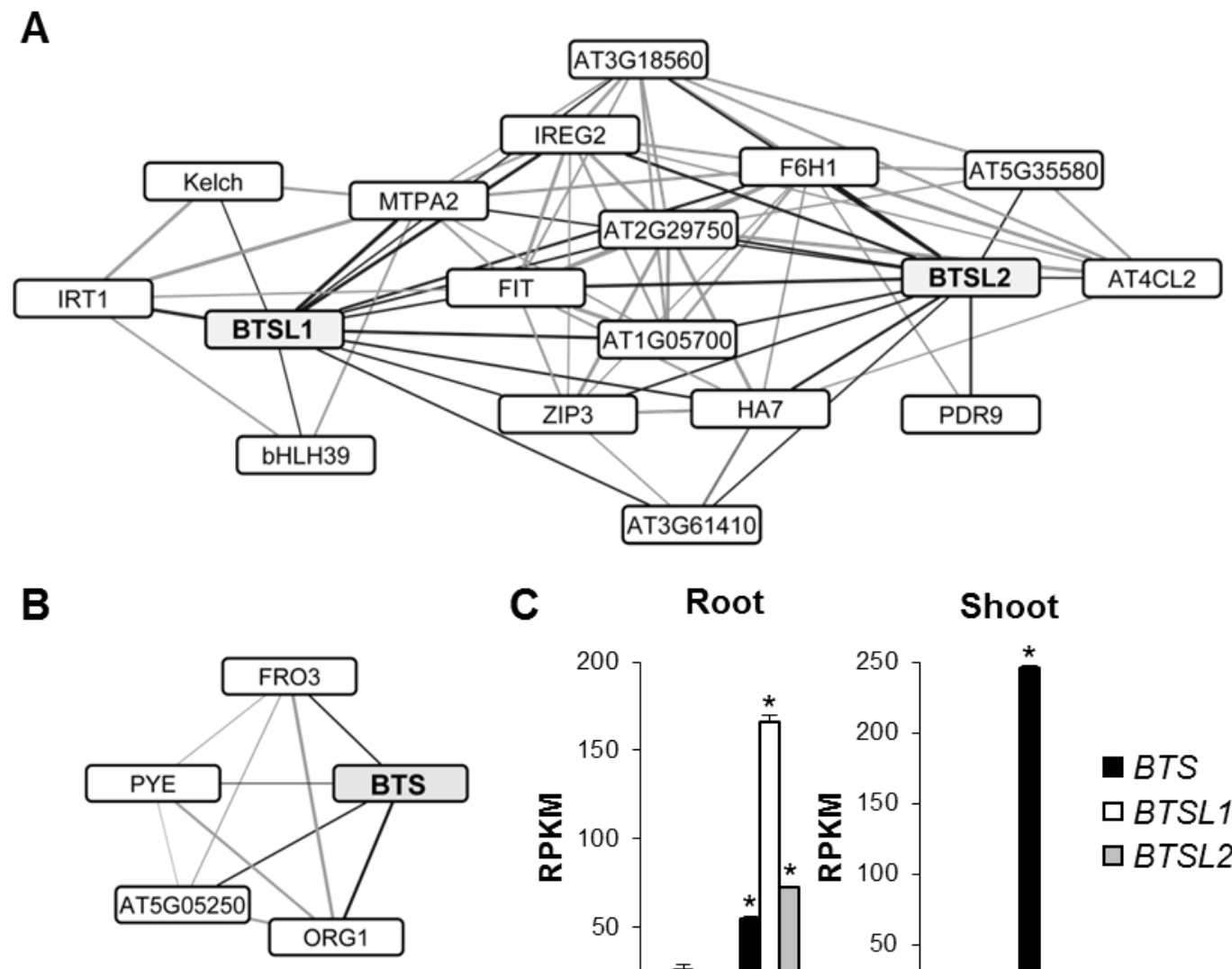

C

Root

Shoot

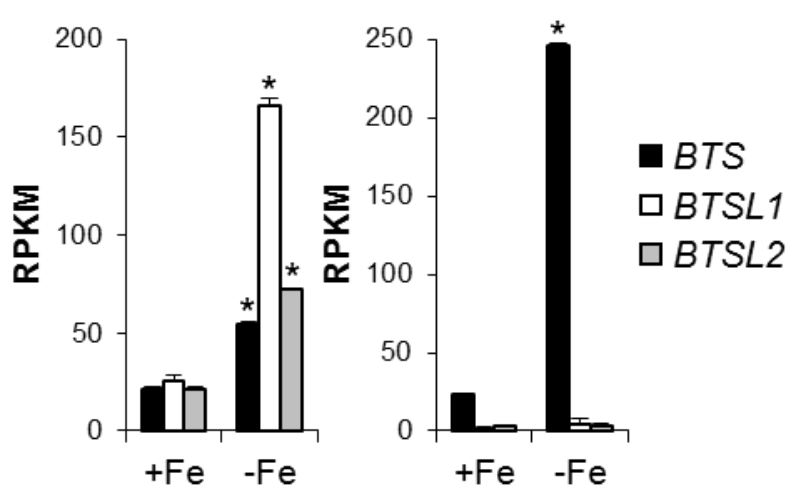

Figure 1. BTSL and BTS are part of different gene expression networks.

(A, B) Co-expression analysis of Fe-responsive genes that are co-regulated with (A) BTSL1 and BTSL2 in roots, and (B) BTS in shoots. The edge thickness relate to the Pearson's correlation coefficient. See Supplemental Table S2 for descriptions of all genes and numerical values.

(C) Expression levels of BTS, BTSL1 and BTSL2 in roots and in shoots under Fe sufficient $(+\mathrm{Fe})$ and $\mathrm{Fe}$ deficient conditions $(-\mathrm{Fe})$. Data were obtained by RNAseq as described in Rodriguez-Celma et al., (2013). RPKM, Reads Per Kilobase Million. The bars represent the mean of $n=3$ biological replicates $\times 10$ seedlings $\pm \operatorname{SD}\left({ }^{*} p<0.05\right.$ using a two-tailed $t$-test).

\section{BTSL1 and BTSL2 proteins are specific to dicotyledonous plants}

To study the evolutionary relationship between BTS and BTSL, we performed a phylogenetic analysis of homologous protein sequences across the tree of life. As noted previously (Kobayashi et al., 2013; Matthiadis \& Long, 2016) homologs containing more than one hemerythrin $(\mathrm{Hr})$ domain fused to a RING-domain E3 ligase are found only among plant species, including mosses. Interestingly, Arabidopsis BTSL1 and BTSL2 are in a separate clade unique to dicotyledonous species (Figure 2A).

BTSL1/2 proteins have two predicted $\mathrm{Hr}$ domains and a CHY/RING Zn-finger domain (Kobayashi et al., 2013). The two Hr domains of BTSL1/2 correspond to the first and third $\mathrm{Hr}$ 
domain in BTS. Further inspection of the amino acid sequence of BTSL1 and 2 revealed a third putative $\mathrm{Hr}$ domain (Figure $2 \mathrm{~B}$ ), not recognized by domain searching algorithms. Alignment of the individual $\mathrm{Hr}$ domains of the three proteins showed that in the gap between the two $\mathrm{Hr}$ domains of BTSL1 and 2 the amino acid sequence is highly homologous to the other $\mathrm{Hr}$ sequences, but lacks the four conserved histidine residues found in the canonical $\mathrm{Hr}$ domains. This domain is still predicted to form a four $\alpha$-helical bundle typical of hemerythrins (Supplementary Figure S2) and additional histidines and acidic residues are present which may function as ligands for Fe. The C-terminal $\mathrm{Zn}$ finger domain is highly conserved, with $80 \%$ amino acid identity between BTSL1 and BTSL2, and 65\% identity with BTS. This domain consists of a unique arrangement of Zn-fingers, a CHY/RING-type Zn finger domain found in the mammalian E3 ligase Pirh2 / RCHY1 (Leng et al., 2003) and in four other E3 ligases in Arabidopsis (Lee and Seo, 2016).

A

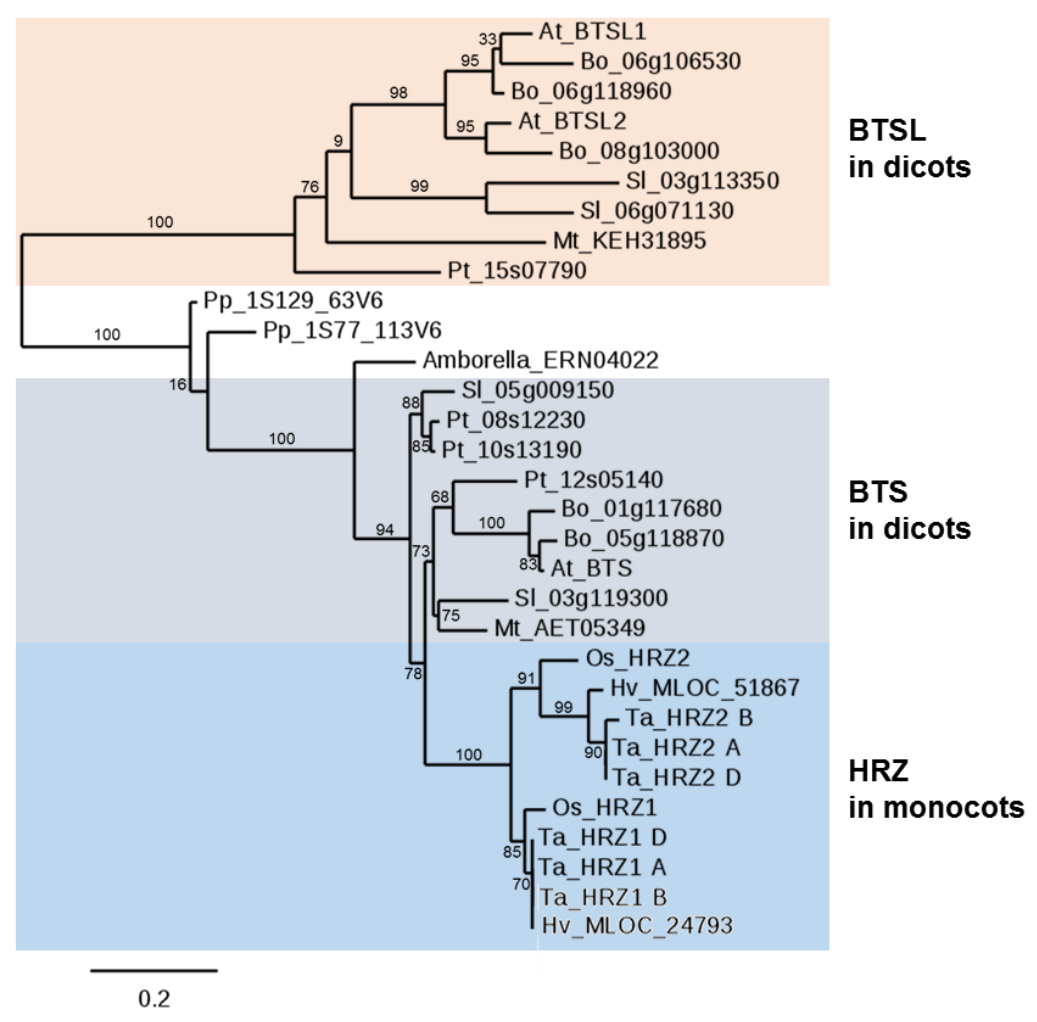

Figure 2. BTSL are uniquely found in dicotyledonous plants.

(A) Phylogenetic tree of BTS homologs from selected plant species. Sequences were found by BLASTing the amino acid sequences of Arabidopsis BTS, BTSL1, BTSL2 and rice HRZ1 in Ensembl Plants (http://plants.ensembl.org). Species used: Amborella, Amborella trichocarpa; At, Arabidopsis thaliana; Bo, Brassica oleracea; $\mathrm{Hv}$, Hordeum vulgare; Mt, Medicago truncatula; Os, Oryza sativa; $\mathrm{Pp}$, Physcomitrella patens; $\mathrm{Pt}$, Populus trichocarpa; SI, Solanum lycopersicum; Ta, Triticum aestivum. Numbers next to branches indicate bootstrapping values for 100 replications. The scale bar indicates the rate of evolutionary change expressed as number of amino acid substitutions per site. (B) Domain organization of BTSL, BTS and HRZ proteins.

B

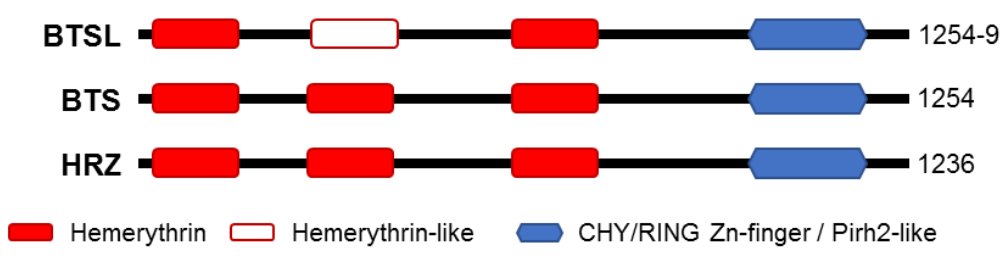




\section{$B T S L 1$ and BTSL2 promoters are activated by Fe deficiency in specific root tissues}

To investigate where in the roots and in which cell types BTSL1 and BTSL2 are expressed, the promoter regions of these genes were cloned into the vector pBGWFS7 upstream of an eGFP-GUS fusion reporter gene (Karimi et al., 2002). For the BTSL1 promoter we used the intergenic region of $880 \mathrm{nt}$ upstream of the ATG start codon. In the case of BTSL2 the intergenic region was $\sim 5 \mathrm{~kb}$, and therefore we decided to use only $2097 \mathrm{nt}$ upstream of the ATG start codon. Seeds were germinated on agar plates with minimum salts (see materials and methods section) supplemented either with $50 \mu \mathrm{M}$ FeEDTA (+Fe) or with $100 \mu \mathrm{M}$ ferrozine to effectively deplete $\mathrm{Fe}(-\mathrm{Fe})$. After 5 days of growth seedlings were analysed for GFP expression. Aside from some autofluorescence in the empty seed coat, no GFP fluorescence was observed in any part of the seedling under Fe sufficient conditions (+Fe). However, under Fe deficiency (-Fe), GFP was highly induced in the roots (Figure $3 \mathrm{~A}$ ), in agreement with RNAseq expression data (Figure 1C). At this early growth stage, GFP expression was similar in the BTSL1 and BTSL2 promoter constructs. In older seedlings that had developed lateral roots, the expression pattern of the BTSL1 and BTSL2 promoters became different: GUS staining showed that BTSL1 is expressed in the upper half of the root under Fe deficiency, whereas BTSL2 is expressed in the lower half of the root, predominantly in the differentiation zone (Figure 3B). BTSL1 and BTSL2 promoter-GUS activity was also found in the root cap (columella), but not in the root meristem or elongation zone. Cross-sections of the GUSstained roots showed that both promoters are induced in the epidermis, including root hairs, and cortex cells. BTSL2 was also expressed in the endodermis and stele in the differentiation zone (Figure 3B). The expression patterns of BTSL1 and BTSL2 contrast with the pattern observed for BTS. BTS promoter-GUS expression showed little promoter activity in the epidermis and cortex, but was strongly upregulated in the stele in response to Fe deficiency (Selote et al., 2015). Moreover, BTS is expressed in embryos which lack BTSL1 and BTSL2 promoter activity (Supplementary Figure S3).

In summary, the BTSL1 and BTSL2 promoters are activated by Fe deficiency in the upper and lower part of the root, respectively. Both genes are expressed in epidermis, cortex and columella cells. 
A

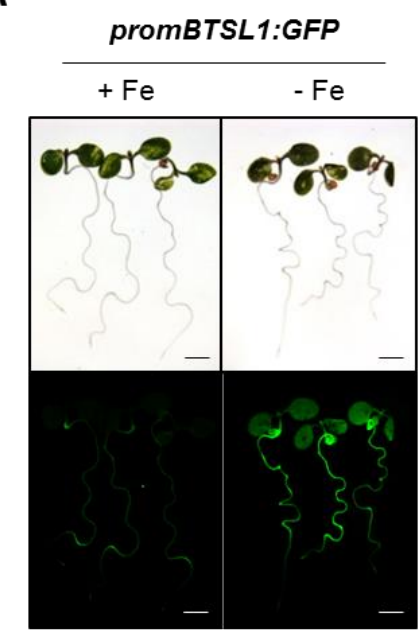

B

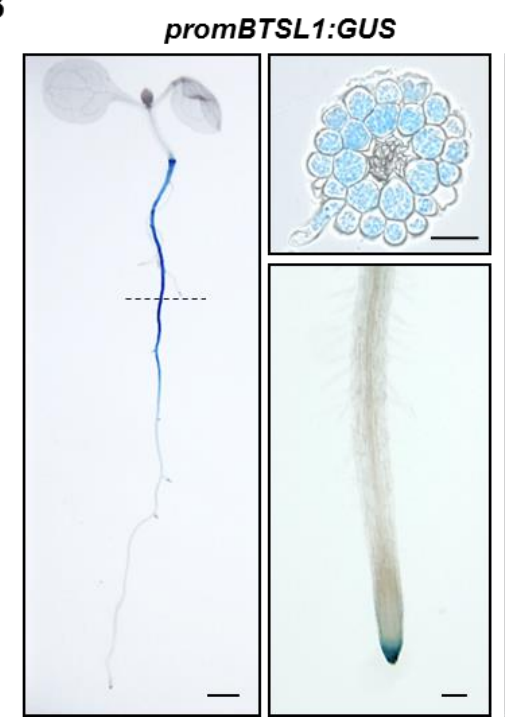

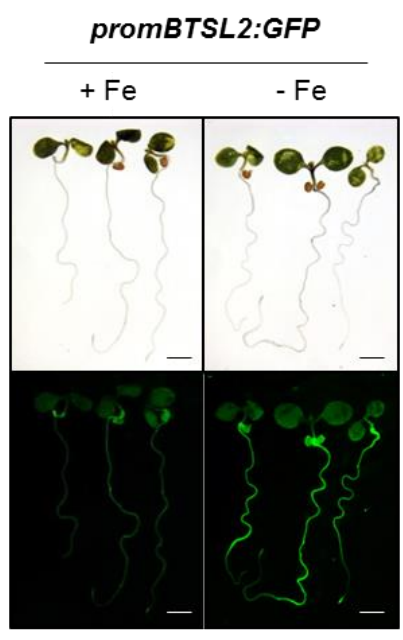

promBTSL2:GUS

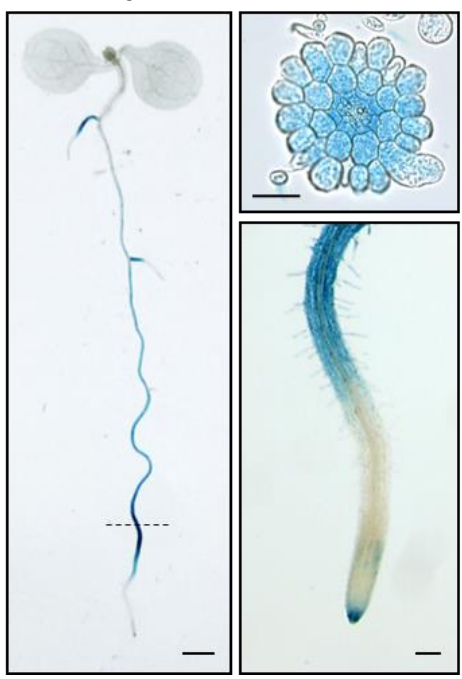

Figure 3. Promoter activity of BTSL1 and $B T S L 2$ in Fe-deficient roots.

Promoter sequences of BTSL1 (-880 nt) and BTSL2 (-2097 nt) were inserted upstream of eGFP-GUS and the constructs were stably expressed in wildtype Arabidopsis.

(A) GFP fluorescence in seedlings grown on medium with $50 \mu \mathrm{M}$ FeEDTA (+Fe) or with $100 \mu \mathrm{M}$ ferrozine to deplete $\mathrm{Fe}$ (-

$\mathrm{Fe}$ ), as indicated, for 6 days. Scale bar is $1 \mathrm{~mm}$.

(B) GUS activity staining in seedlings grown on medium depleted of $\mathrm{Fe}(100$ $\mu \mathrm{M}$ ferrozine) for 8 days. Scale bar is 0.5 $\mathrm{mm}$ for whole seedlings, $100 \mu \mathrm{m}$ for a close-up of the root tip, and $50 \mu \mathrm{m}$ for root cross sections.

Images are representative of 3 independent lines for each promoter construct.

\section{$B T S L 1$ and BTSL2 have partially redundant functions in maintaining Fe homeostasis}

To characterise the function of BTSL in Fe homeostasis, T-DNA insertion lines were obtained from the Nottingham Arabidopsis Stock Centre. Three independent mutant lines were selected for BTSL1 (SALK_015054, SALK_032464 and SALK_117926; named bts/1-1, bts/1-2 and $b t s / 1-3$, respectively), but only one line with a T-DNA insertion in the coding sequence was available for BTSL2 (SALK_048470; bts/2-2) (Figure 4A). The bts/1-1 was described as bts/1 in Hindt et al., (2017) and the bts/2 allele used by the same authors has an insertion in the promoter. Homozygous lines were selected by PCR, T-DNA insertion sites were confirmed by sequencing, and transcript levels were assessed by RT-qPCR. The expression of BTSL1 in roots under standard and Fe deficient conditions was virtually abolished in the bts/1-1 and bts/1-3 alleles, but residual expression remained in bts/1-2 (Figure 4B). Disruption of the $B T S L 1$ gene led to a 2-fold upregulation of BTSL2 expression under Fe deficiency (Figure 
4C). The bts/2-2 mutant lacked detectable levels of BTSL2 transcript (Figure 4C), while BTSL1 expression was $\sim 3$-fold higher than wild type lacking Fe (Figure 4B). Double knock-out lines were produced by crossing bts/1-1 and bts/1-3 with the bts/2-2 line. The bts/1-1 bts/2-2 mutant was genetically complemented by either BTSL1 or BTSL2 (see below).

Mutant lines were tested for phenotypes on a range of Fe concentrations. Seedlings were germinated and grown on medium with $50 \mu \mathrm{M}$ FeEDTA for 10 days, followed by 4 days on plates with ferrozine (-Fe), or 4 days on Fe sufficient medium (50 $\mu \mathrm{M}$ FeEDTA). The effect of toxic concentrations of Fe was tested by growing the seedlings on plates with 200 or $500 \mu \mathrm{M}$ FeEDTA for 14 days. Single insertion lines showed no obvious phenotype under any of the conditions tested. On the other hand, the bts/1-1 bts/2-2 double mutant line showed subtle but noticeable phenotypes under conditions of both Fe deficiency and Fe excess (Figure 4D). After 4 days without $\mathrm{Fe}$, the bts/ double mutant appeared less chlorotic than wild type, which was confirmed by chlorophyll measurements (Figure 4E). The bts/2-2 mutant allele also retained some chlorophyll, suggesting that BTSL1 and BTSL2 are not fully redundant. In the presence of excess $\mathrm{Fe}$, growth of the bts/ double mutant was significantly more impaired than wild type or the single mutants (Figure 4D; Supplementary Figure S4). Quantification of Fe levels in shoots showed a 2-fold accumulation of this metal in bts/plants grown on excess Fe (Figure $4 \mathrm{~F}$ ), indicating that this might be the cause of the observed growth impairment. 
A

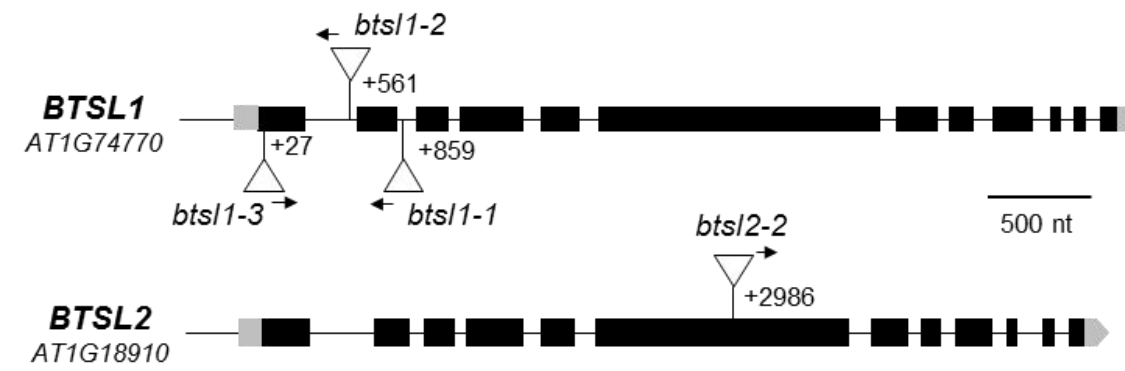

B



C

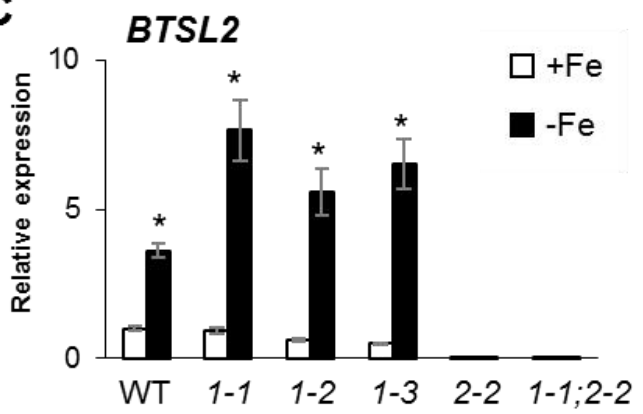

D

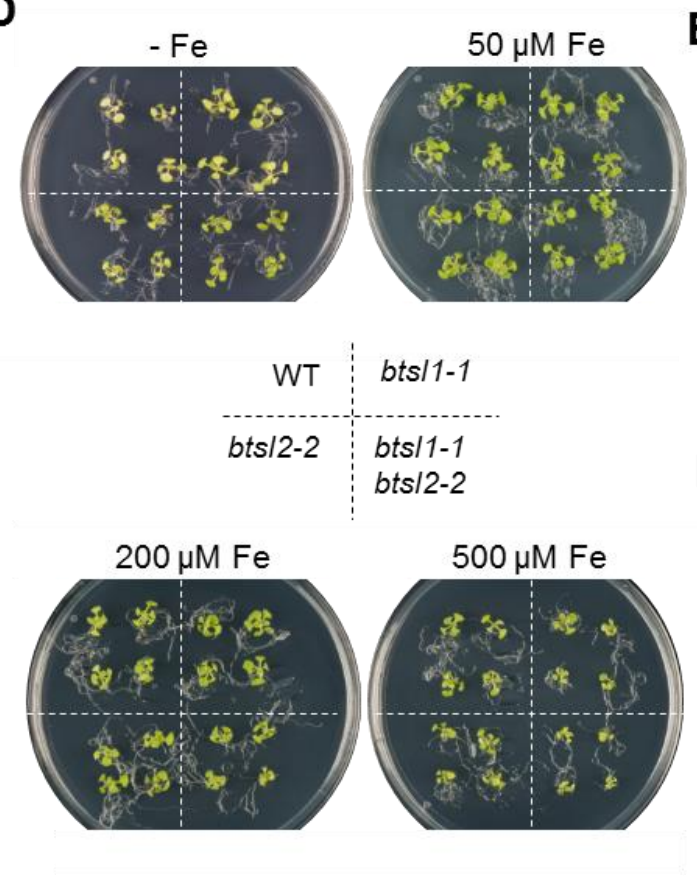

E

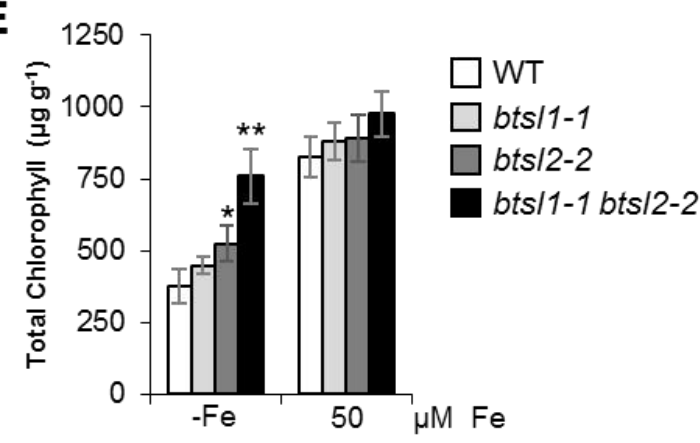

$\mathbf{F}$



Figure 4. BTSL1 and BTSL2 have redundant functions in Fe homeostasis.

(A) Schematic representation of the BTSL1 and BTSL2 genes and mutant alleles. Exons are in black, 5' and 3'-UTRs in grey. T-DNA insertions are indicated by triangles: bts/1-1, SALK_015054; bts/1-2, SALK_032464; bts/1-3, SALK_117926; bts/2-2, SALK_048470. Arrows indicate the left border primer used for genotyping.

(B, C) Transcript levels of BTSL1 (B) and BTSL2 (C) in wild-type and mutant alleles under standard (+Fe, 50 $\mu \mathrm{M}$ FeEDTA) and Fe-deficient conditions (-Fe, $100 \mu \mathrm{M}$ ferrozine). ( ${ }^{*} \mathrm{p}<0.05$ using a two-tailed $t$-test)

(D) Growth of wild-type and btsl alleles on medium with varying Fe concentrations. Seeds were germinated on medium with $50 \mu \mathrm{M}$ FeEDTA, and, after 10 days, transferred to medium depleted of $\mathrm{Fe}$ using $100 \mu \mathrm{M}$ ferrozine $(-\mathrm{Fe})$ or $50 \mu \mathrm{M}$ FeEDTA; or grown in the presence of 200 or $500 \mu \mathrm{M}$ FeEDTA for 14 days.

(E, F) Chlorophyll concentration (E) under - Fe and control conditions, and iron concentration in shoots $(F)$ from wild type (WT) and bts/ mutant alleles, grown under control and Fe toxicity conditions as in (D). The bars represent the mean of $\mathrm{n}=3$ biological replicates $\times 10$ seedlings $\pm \mathrm{SD}\left({ }^{*} p<0.05 ;{ }^{* *} \mathrm{p}<0.01\right.$ using a two-tailed $t$ test). 


\section{BTSL1 and BTSL2 limit Fe uptake after a period of Fe deficiency}

Because BTSL1 and BTSL2 are only expressed in Fe-deficient roots, but phenotypes were seen under Fe excess, we tested whether the genes have a specific function in the transition from Fe deficiency to Fe sufficiency. Wild-type and bts/ double mutant seedlings were grown up on medium with $\mathrm{Fe}$ ( $50 \mu \mathrm{M}$ FeEDTA) for ten days and then transferred to medium without Fe but with ferrozine for 3 days to induce the Fe deficiency response. After that, plants were resupplied with Fe by transferring them back to Fe sufficient conditions, and then sampled after 3 days. In the control treatment, seedlings were always grown on $50 \mu \mathrm{M}$ Fe for $10+3+$ 3 days, with transfers to fresh medium. A diagram of the treatments and time points of sampling is presented in Figure 5A. For comparison, we also grew the brutus-1 (bts-1) mutant line (Selote et al., 2015) under the same conditions. bts-1 is a viable allele that lacks mRNA induction under Fe deficiency.

A

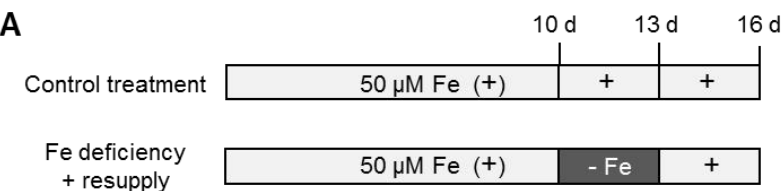

B

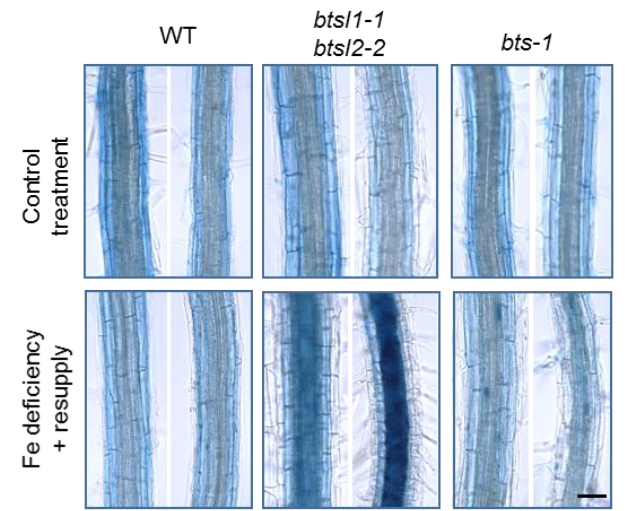

C

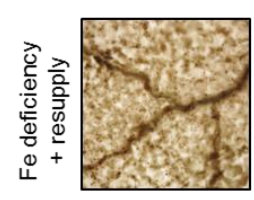

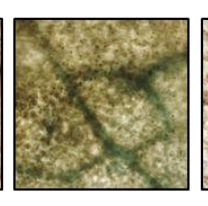

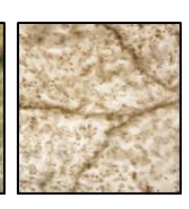

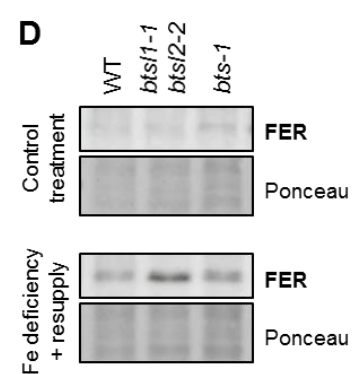



-WT

口bts/1-1 bts/2-2

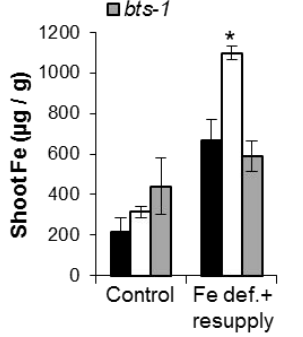

Figure 5. The btsl double mutant hyperaccumulates $\mathrm{Fe}$ after a period of deficiency.

(A) Diagram of the $\mathrm{Fe}$ treatments used in this study. Seedlings were germinated on agar plates with $50 \mu \mathrm{M}$ FeEDTA. On day 10 , seedlings were transferred to a new plate (control treatment) or to medium depleted of $\mathrm{Fe}(100 \mu \mathrm{M}$ ferrozine). After 3 days, seedlings were transferred back to medium with $50 \mu \mathrm{M}$ FeEDTA. Samples were taken at the end of the treatment.

(B) Perls' Prussian Blue staining for $\mathrm{Fe}$ in roots in wild-type, bts/1-1 bts/2-2 double mutant and bts-1 seedlings following control (top) and Fe deficiencyresupply treatments (bottom) according to the diagram in (A). The images show a section of the differentiation zone. Scale bar is $50 \mu \mathrm{m}$.

(C) Perls' Prussian Blue staining of leaves after the $\mathrm{Fe}$ deficiency-resupply treatment. The images are a close-up of the adaxial leaf surface with veins. Scale bar is $75 \mu \mathrm{m}$.

(D) Immunoblot of ferritin (FER) protein levels in root samples of wild type, bts/1-1 bts/2-2 double mutant and bts-1 seedlings after control and resupply treatments.

(E) Quantification of $\mathrm{Fe}$ in the shoots of wild-type bts/1-1 bts/2-2 double mutant and bts-1 seedlings after control and $\mathrm{Fe}$ deficiency-resupply treatments. Bars represent the mean of 3 biological replicates of 10 pooled plants each \pm SD $\left({ }^{*} p<0.05\right.$ using a two-tailed $t$-test).

None of the genotypes showed chlorosis or growth impairment after the Fe deficiency and resupply treatment. However, the bts/double mutant accumulated large amounts of Fe in the central core of the root and in leaf veins as shown by Perls' staining (Figure 5B, C). Iron did not accumulate when mutants were grown continuously on $50 \mu \mathrm{M} \mathrm{Fe}$. bts-1 mutants behaved as wild-type plants and did not show increased Perls' staining in any part of the root or leaves after the Fe deficiency-resupply treatment.

To gain further insight into the management of the Fe influx, we analysed the levels of ferritin protein, which functions as a temporary Fe store in Arabidopsis roots and leaves (Ravet et al., 
2009). Ferritin protein levels followed the same trend as observed with Fe staining, with ferritin accumulating only in the bts/ double mutant after the Fe deficiency-resupply treatment (Figure 5D).

When we measured the Fe content of the shoots, we found that wild-type plants, when subjected to deficiency and resupply, contained 3-fold more Fe compared to continuous growth on $50 \mu \mathrm{M} F e$ (Figure $5 \mathrm{E}$ ). It is likely that upregulation of the Fe uptake machinery during Fe deficiency leads to a sudden influx of Fe when this is resupplied. In wild-type seedlings, the increased Fe levels do not seem to be toxic as there is no growth impairment and $\mathrm{Fe}$ is evenly distributed (Figure 5B, C). In the bts/ double mutant, the Fe content of shoots increased more than 5-fold after the deficiency-resupply treatment relative to the control conditions (Figure 5E), reaching similar levels to those observed in plants grown continuously with 500 $\mu \mathrm{M}$ Fe (Figure 4F). In contrast to the bts/ double mutant, bts-1 seedlings had a similar Fe content to wild type after the Fe deficiency-resupply treatment (Figure 5E).

In summary, our data show that the bts/ double mutant is unable to adapt to changes in $\mathrm{Fe}$ supply, accumulating toxic concentrations of Fe. Moreover, the lack of Fe accumulation in bts1 line under these conditions suggests that BTSLs and BTS have different functions or act in different tissues.

\section{The bts/1-1 bts/2-2 double mutant fails to switch off the Fe deficiency response}

Next, we investigated the levels of FRO2 and IRT1, two key players in Fe uptake, during Fe deficiency and resupply in the wild-type and bts/ double mutant. We followed the same treatment described above, and plants were sampled before the resupply from the control (+Fe) and Fe deficiency treatment (-Fe), and daily after Fe resupply (1, 2 and $3 \mathrm{~d}$ Fe resupply) (Figure 6A). Wild-type seedlings were able to regulate FRO2 enzyme activity as expected, showing a $\sim 6$-fold induction in response to Fe deficiency and returning down to basal levels one day after Fe resupply (Figure 6A). In bts/ double mutant seedlings FRO2 enzyme activity remained high after Fe resupply: the activity was 4-fold induced after one day, 3-fold induced after two days and it was still nearly double the basal wild-type levels after three days (Figure 6A). The single insertion bts/1-1 and bts-1 mutant lines behaved like the wild type, but bts/2-2 showed a slight delay in switching off FRO2 activity (Supplementary Figure S5A). The sustained induction of $\mathrm{FRO} 2$ activity in the bts/ double mutant was restored by expressing either 35S:BTSL-YFP or 35S:BTSL2-GFP (Supplementary Figure S5B).

IRT1 protein levels in wild-type plants were strongly increased under Fe deficiency, and were nearly below detection after one day of Fe resupply. In the bts/ double mutant, IRT1 was not detected under standard conditions $(+\mathrm{Fe})$, but after three days of Fe deficiency was present 
at much higher levels than in wild-type roots. Upon Fe resupply, the IRT1 protein levels fluctuated but remained high overall (Figure 6B). The sustained presence of FRO2 and IRT1 would explain the increased Fe accumulation in the bts/ double mutant following the Fedeficiency-resupply treatment.

A

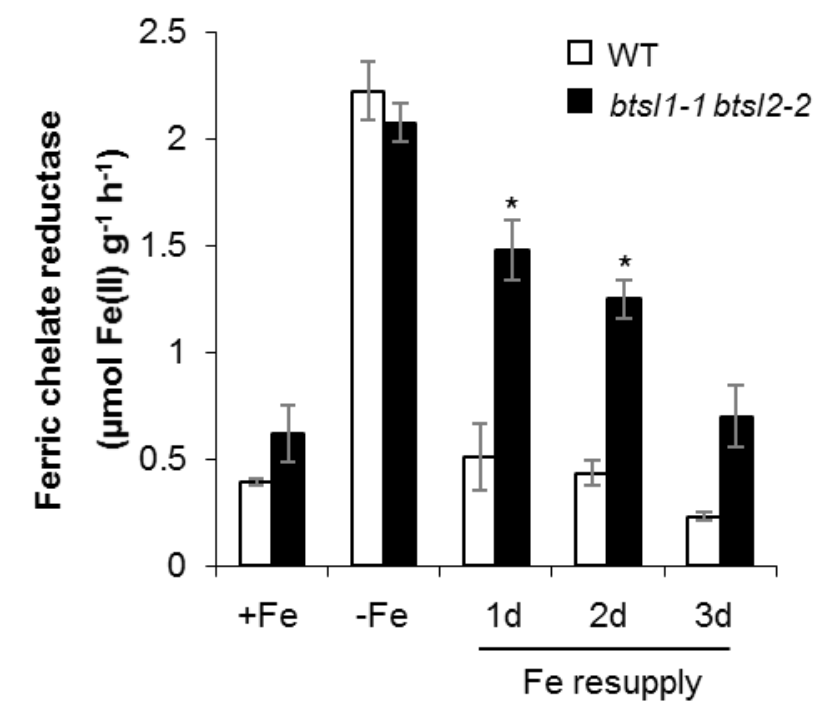

B

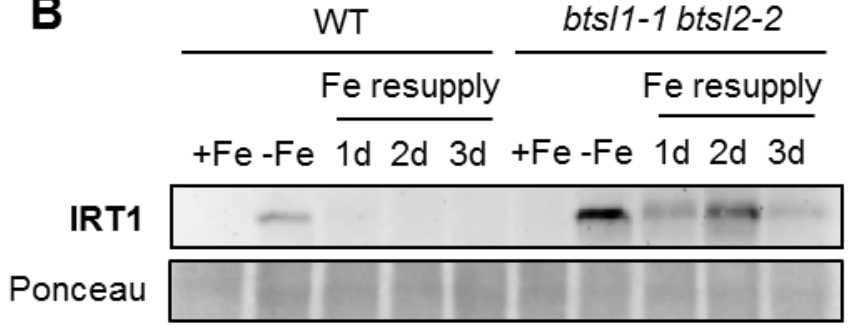

C

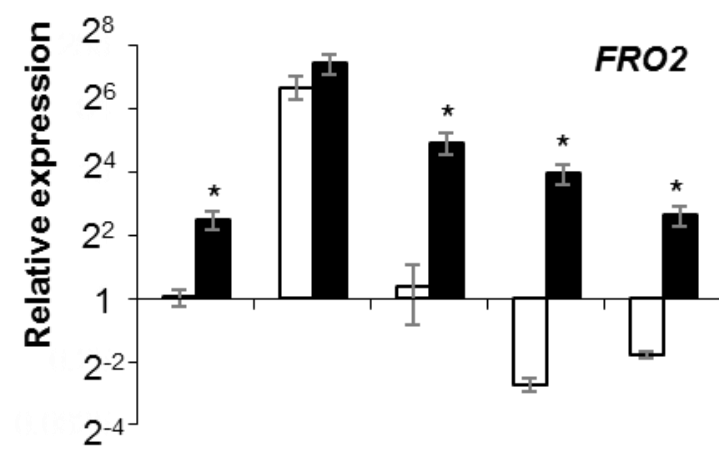

D<smiles>[AlH2]</smiles>

bts $11-1$ bts $/ 2-2$

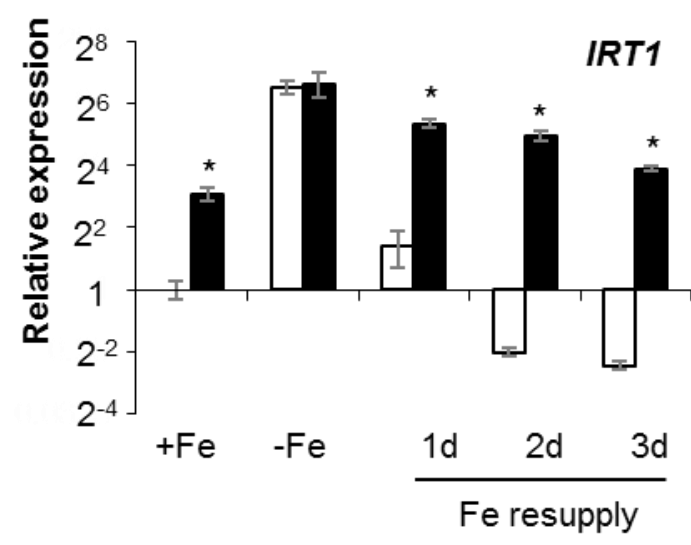

Figure 6. The bts/ double mutant maintains FRO2 and IRT1 upon Fe resupply.

(A) Ferric chelate reductase activity of wild-type and bts/1-1 bts/2-2 mutants. Bars represent the mean of 3 biological replicates ( $n=5$ seedlings/assay) $\pm \mathrm{SD}\left({ }^{*} \mathrm{p}<0.05\right.$ using a two-tailed $t$-test).

(B) Immunoblot of IRT1 protein levels in root samples in the Fe resupply treatment.

(C, D) Expression of FRO2 (C) and IRT1 (D) in roots from wild-type and bts/1-1 bts/2-2 seedlings determined by quantitative RT-PCR. All values are relative to wild type $+F e$. Values are the mean of 3 biological replicates $\pm S E$ ( ${ }^{*} p<0.05$ using a twotailed $t$-test).

To investigate which step of FRO2 and IRT1 expression is misregulated, we analysed transcript levels of $F R O 2$ and $I R T 1$ by RT-qPCR. Interestingly, under control conditions $(+\mathrm{Fe})$, FRO2 and IRT1 transcripts levels were already increased 5 to 8-fold, respectively, in the btsI double mutant compared to wild type (Figure 6C, D), although no increase in enzymatic activity or protein levels was observed (Figure 6A, B). After three days of Fe deficiency, transcription of $F R O 2$ and $I R T 1$ was strongly upregulated by a similar magnitude in both mutant and in wild type. However, upon Fe resupply, FRO2 and IRT1 transcript levels remained high in the bts/ double mutant, matching the sustained FRO2 activity and IRT1 protein levels observed (Figure 
6C). In contrast, in wild type, FRO2 and IRT1 transcripts were downregulated on day 2 and 3 after resupply compared to the initial levels (Figure 6C). Taken together, these data show that the transcriptional control of FRO2 and IRT1 is altered in the bts/ double mutant and is disconnected from the Fe concentration in the medium.

\section{The transcriptional regulator FIT-bHLH38/39 is mis-regulated in the bts/ double mutant}

The transcription of FRO2 and IRT1 is directly regulated by FIT together with one of four partially redundant bHLH transcription factors (bHLH38, bHLH39, bHLH100, bHLH101). To investigate if FIT is misregulated, its protein and transcript levels were followed in wild-type and mutant plants subjected to the Fe deficiency-resupply treatment. Under control conditions, FIT was not detected by protein blot analysis in either wild-type or bts/1-1 bts/2-2. FIT protein levels were strongly induced after three days of Fe deficiency in wild-type seedlings, and even more so in bts/ double mutant seedlings ( 3-fold more than wild type, Figure 7A). Upon Fe resupply, FIT protein levels fell below the limit of detection in wild type, but were partially sustained in bts/ roots, which could explain the increase in FRO2 and IRT1 transcripts (Figure $6 \mathrm{C})$. In contrast, the transcriptional regulation of $F I T$ was similar in wild-type and bts/ seedlings (Figure 7B). This suggests than the protein levels of FIT may be directly or indirectly controlled by BTSL1 and/or BTSL2 under Fe deficiency, as well as upon Fe resupply.

As already stated, FIT functions as a heterodimer, therefore we tested $b H L H 38$ and $b H L H 39$ transcript levels as a representative of all four redundant bHLH transcription factors from the subgroup lb. Interestingly, under control conditions, $b H L H 38 / 39$ transcripts levels were increased 8-fold in the bts/ double mutant compared to wild type (Figure 7B). After three days of Fe deficiency, the upregulation was similar in both genotypes, but upon Fe resupply, $b H L H 38 / 39$ transcript levels remained high in the bts/ double mutant, matching the patterns observed for FRO2 and IRT1 transcripts (Figure 7B). In wild type plants, as soon as day 1 after resupply $b H L H 38 / 39$ transcripts were downregulated below initial levels (Figure 7C).

In summary, our data show that BTSL1 and 2 are required for FIT protein turnover, and for repressing transcription of $b H L H 38 / 39$. 


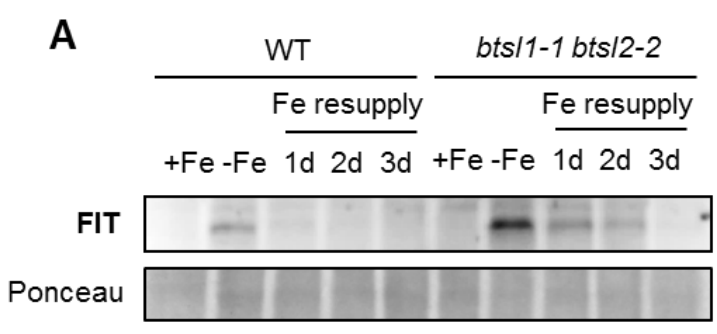

B

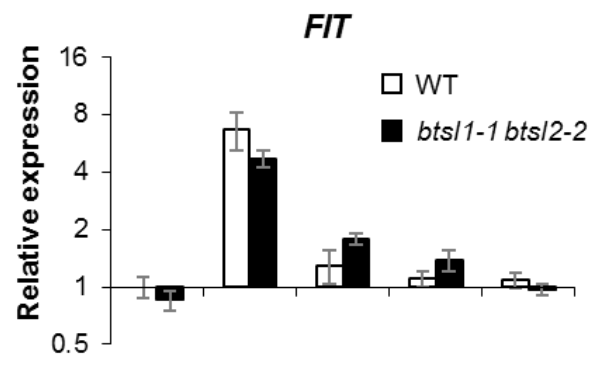

bHLH38
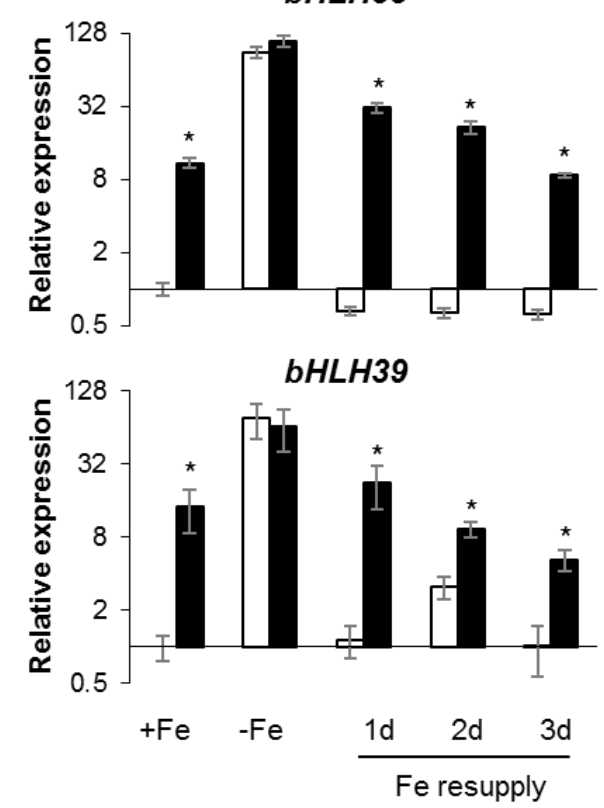

Figure 7. FIT protein and $b H L H 38, b H L H 39$ transcript levels are misregulated in the btsI double mutant upon Fe resupply.

(A) Immunoblot of FIT protein levels in root samples of the Fe deficiency-resupply treatment.

(B) Expression of FIT, bHLH38 and bHLH39 in roots from wild-type and $b t s / 1-1 \mathrm{bts} / 2-2$ seedlings determined by quantitative RT-PCR. All values are relative to wild type $+\mathrm{Fe}$. Values are the mean of 3 biological replicates $\pm S E\left({ }^{\star} p<0.05\right.$ using a twotailed $t$-test).

\section{BTSL1 and BTSL2 interact with FIT in vitro}

Next, we tested if BTSL proteins can physically interact with FIT, a prerequisite for a direct target of ubiquitin-mediated protein degradation. A standard yeast 2-hybrid approach was not possible because of self-activation by the individual proteins. Therefore, we opted for an in vitro approach. The C-terminal CHY/RING Zn-finger domain of BTSL1 or BTSL2 was fused to MBP (maltose binding protein) for stabilization, and expressed in E. coli. FIT was also produced in E. coli with a Myc tag at the C-terminus. bHLH39:Myc and bHLH40:Myc were produced as controls. The protein interactions were studied by Far-Western blot analysis (Figure $8 \mathrm{~A}$ ) to overcome issues with the stability of the transcription factors. E. coli extracts with FIT, bHLH39 or bHLH40 were separated by SDS-PAGE and immobilized on nitrocellulose 
membrane. Immuno-detection of the Myc tag confirmed the presence and protein levels of FIT, bHLH39 and bHLH40 (Figure 8B and Supplementary Figure S6, left panels). Purified MBP, MBP:FIT or MBP:BTSL proteins were incubated with the membrane, followed by antibody detection of the MBP tag. MBP:FIT bound strongly to bHLH39 (35 kDa band) and weakly to itself ( $55 \mathrm{kDa}$ band), as previously reported (Yuan et al 2008). The immuno-signals were clearly separated from an aspecific band in the MBP control. MBP:BTSL1c and MBP:BTSL1c bound to FIT, but not to bHLH39. A separate set of experiments showed that the BTSL proteins also did not interact with bHLH40 (Supplementary Figure S6), better known as the transcription factor INDEHISCENT involved in abscission but unrelated to iron homeostasis (Liljegren et al., 2004). These data suggest that both BTSL1 and BTSL2 bind FIT to target it for degradation.
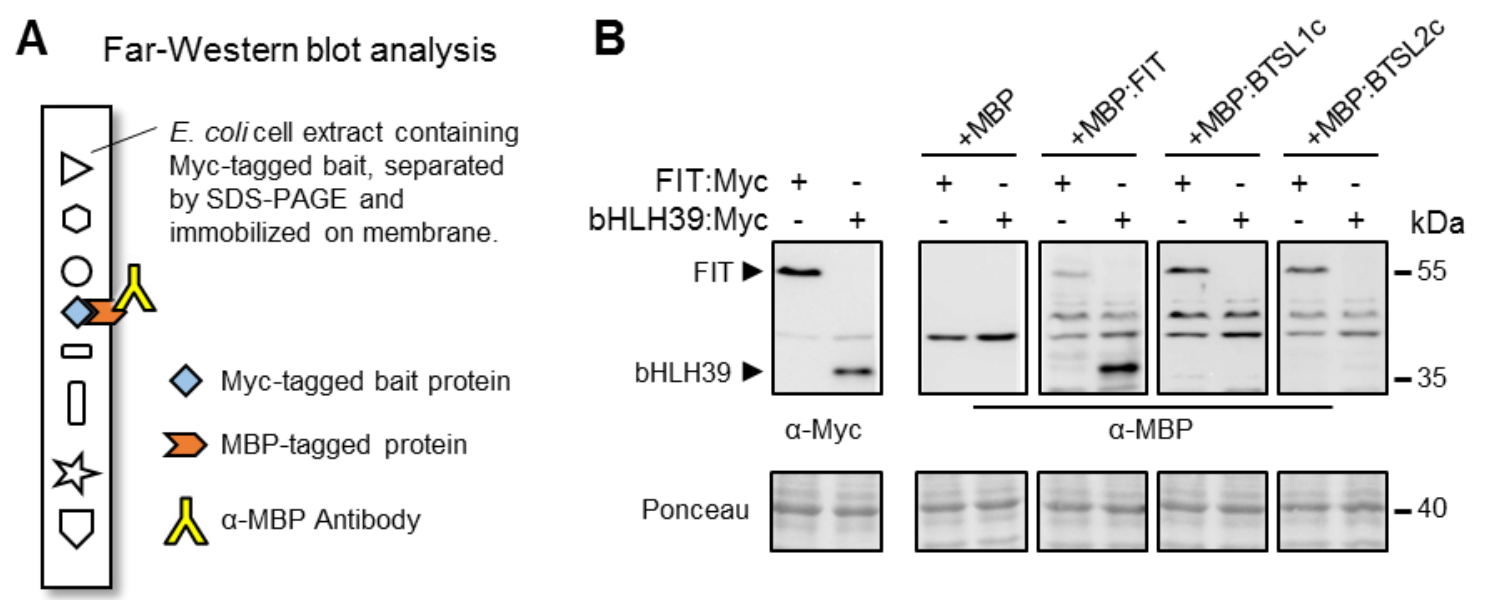

Figure 8. The CHY/RING Zn finger domain of BTSL proteins interact with FIT.

(A) Diagram of Far-Western blot analysis. Bait proteins with a Myc-tag were produced in E. coli and protein extracts separated by SDS-PAGE gel followed by transfer to nitrocellulose membrane. Blots were incubated with purified MBP-tagged proteins of interest, then labelled with anti-MBP antibodies.

(B) Far-Western blots analysis showing direct protein interactions between BTSL proteins and FIT. Bacterial protein extracts with FIT:Myc and bHLH39:Myc were immobilized on nitrocellulose, and the blots were incubated with recombinant MBP, MBP:FIT and the C-terminal domains of BTSL1 and BTSL2, fused to MBP (MBP:BTSL1c and MBP:BTSL2c). FIT is known to form a heterodimer with bHLH39. Ponceau staining shows equal protein loading. 


\section{DISCUSSION}

In this study, we show that BTSL1 and BTSL2 have a clearly distinct function from BTS, and are not simply redundant paralogs. BTSL genes have evolved specifically in dicotyledonous plants, they are root specific and co-regulated with the Fe uptake machinery. We show that the partially redundant BTSL proteins negatively control Fe uptake in the plant, which is exacerbated under Fe deficiency-resupply conditions. Under these conditions, BTS does not play any role. A closer look at the expression patterns of the BTSL1, BTSL2 and BTS genes suggest a demarcation by the Casparian strip, an effective barrier against nutrient overload of plants. The two BTSL genes are predominantly expressed in the root epidermis and cortex, whereas BTS is expressed inside the root stele and in the shoot. BTSL2 is also expressed in the stele in the differentiation zone, but this is where the Casparian strip is not yet formed or still permeable (Nasser et al., 2012; Barberon et al., 2016). Moreover, the BTSL2 promoterGUS expression was analysed under Fe deficient conditions, when suberinization of the Casparian strip is suppressed to maximize metal and nutrient uptake (Barberon et al., 2016). We propose a model where BTSL proteins act as a primary defense mechanism against excess Fe uptake in the root. A second defense barrier against Fe overload would be the BTS protein in the stele and leaf tissues, behind the Casparian strip (Figure 9).
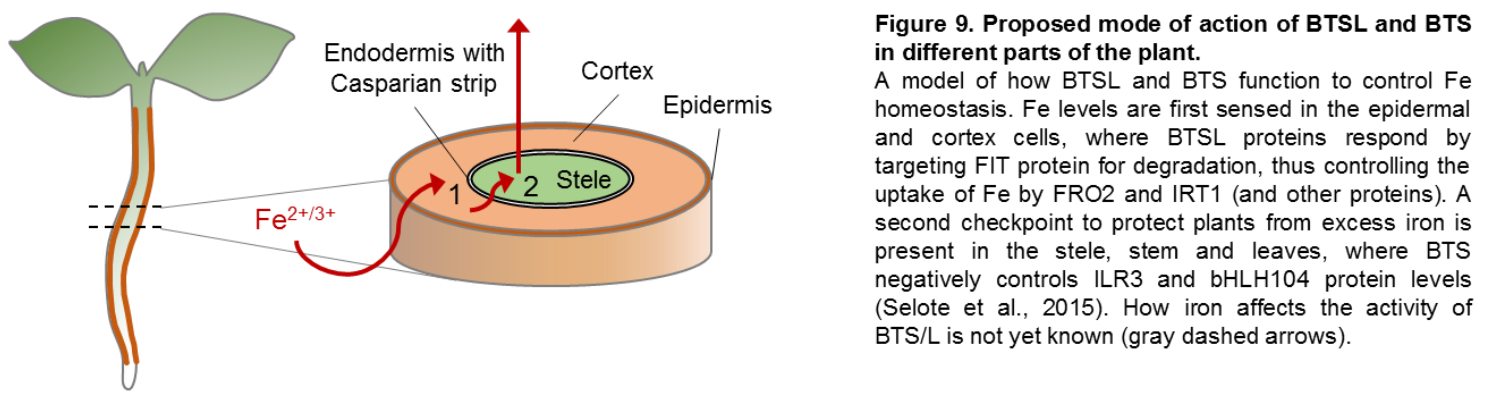

Check point 1: BTSL in the roots

Check point 2: BTS in the stele

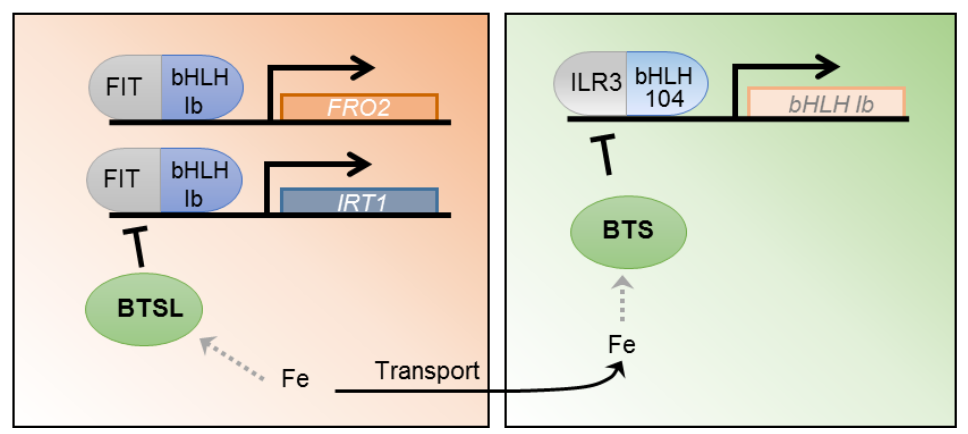

The different functions of BTSL1/2 and BTS suggest that the E3 ligases target different proteins for degradation. Their C-termini are rather unique among E3 ligases, consisting of a combination of CHY/RING-type Zn finger domains, as shown in NMR structures of the 
mammalian Pirh2/RCHY1 homolog (Sheng et al., 2008) which shares $45 \%$ amino acid identity with the $\mathrm{C}$-termini of $\mathrm{BTS} / \mathrm{L}$. Pirh2/RCHY1 binds to the tetrameric form of the $\mathrm{p} 53$ transcription factor and targets it for degradation (Leng et al., 2003; Sheng et al., 2008). In Arabidopsis, 7 proteins share this conserved Pirh2-like domain: AT3G62970, AT5G18650, AT5G25560, AT5G22920; and BTSL1, BTSL2 and BTS. The best characterized is MYB30-INTERACTING E3 LIGASE 1 (MIEL1, AT5G18650), which targets the MYB30 transcription factor for degradation (Lee and Seo, 2016). Thus, it is likely that the unique CHY/RING-finger domain facilitates interaction with transcription factors for ubiquitination and degradation. BTS was found to interact and induce the degradation of the bHLH transcription factors ILR3 and bHLH115 (Selote et al., 2015), but direct ubiquitination of these two putative targets remains to be shown. It has recently been shown that HRZ1, the BTS homolog in rice, targets PRI1 (Positive Regulator of Iron homeostasis1) for degradation (Zhang et al., 2017). The bHLH transcription factor PRI1 is the homolog of Arabidopsis ILR3 and promotes the expression of OsIRO2 and OsIRO3, which are orthologous to $b H L H 38 / 39$ and $P Y E$, respectively (Kobayashi et al., 2013).

Our data suggest that the BTSL proteins target FIT for degradation. Firstly, the expression patterns of FIT and BTSL genes overlap in the epidermis and cortex (Figure 3; Colangelo and Guerinot, 2004; Jakoby et al., 2004). Secondly, FIT protein levels are increased in the btsl double mutant (Figure 7). Thirdly, the C-termini of BTSL1 and BTSL2 interact with FIT in vitro (Figure 8). It has previously been reported that FIT is regulated post-transcriptionally via proteasomal degradation (Sivitz et al., 2011), being turned over in response to ethylene (Lingam et al., 2011). Our data suggest that the BTSL proteins act as the E3 ligases responsible for ubiquitination of FIT which is then degraded by the $26 \mathrm{~S}$ proteosome. At the moment, we cannot rule out that BTSL proteins may have other targets. The misregulation of bHLH38 and bHLH39 expression cannot be explained by lack of FIT protein degradation, because the transcriptional activitators of bHLH38/39 are ILR3 and bHLH104 (Zhang et al., 2015). Either BTSL also target ILR3 and/or bHLH104 for degradation specifically in those root tissues where BTS is not expressed, or mutation of the BTSL genes has an indirect effect on the BTS regulon. A comprehensive study of the direct targets of BTSL and BTS proteins will clarify these questions, although the experimental approaches for determining E3 ligases targets are not straight forward (Iconomou and Saunders, 2016).

Where the Fe status is sensed is still under debate (Kobayashi and Nishizawa, 2014; Gayomba et al., 2015). The observation that BTSL and BTS are expressed in different tissues support the hypothesis that $\mathrm{Fe}$ is sensed independently in different parts of the plant. Our data suggest that BTSLs act as local Fe sensors controlling Fe uptake. FRO2 activity and transcript levels are misregulated in the bts/ double mutant after Fe resupply. The same happens with 
IRT1 protein and transcript levels, that remain high irrespective of the new Fe status. It is interesting to note the fluctuating levels of IRT1 protein after Fe resupply, which could be explained by its own internalization/degradation mechanism (Barberon et al., 2011; Shin et al., 2013). This protective mechanism seems to be disconnected from transcriptional regulation, with decreased levels of detectable IRT1 protein one day after resupply. However, the sustained transcript levels then seem to overrule the post-translational control mechanism, and two days later detectable protein levels are almost as high as under Fe deficiency. On the inside of the Casparian strip, BTS could sense the Fe status in the shoot and control systemic signals that regulate Fe homeostasis. Both local and systemic controls of Fe homeostasis have been reported (Grusak and Pezeshgi, 1996; Schikora and Schmidt, 2001; Vert et al., 2003).

An open question still is of how the BTS/L proteins sense Fe and adjust their E3 ligase activity. The BTS/L proteins have conserved hemerythrin domains that are predicted to bind $2 \mathrm{Fe}$ atoms per domain. Recombinantly expressed HRZ and BTS were found to bind 2 Fe and 1 $5 \mathrm{Zn}$ atoms (Kobayashi et al., 2013; Selote et al., 2015), which is far below the 6 Fe expected for $3 \mathrm{Hr}$ domains and $9 \mathrm{Zn}$ for the CHY/RING-type Zn finger domain. HRZ and BTS proteins seem to be rather unstable, and GFP fusions are not detected (Selote et al., 2015). Removal of the N-terminal domain with the three hemerythrin motifs led to a stable GFP fusion protein, suggesting that this domain regulates the stability of BTS. Based on in vitro data, Selote et al (2015) concluded that BTS protein is stabilized in the absence of Fe bound to the hemerythrin domains. However, this mechanism is the opposite of what is observed for FBXL5, where Fe binding to the $\mathrm{Hr}$ domain stabilized the protein (Salahudeen et al., 2009). The fact that the phenotypes in bts/ mutants are particularly noticeable upon Fe resupply points towards a stabilization of BTSL protein by $\mathrm{Fe}$, in a mechanism analogous to that of FBXL5. However, the changes in protein turnover in FIT and IRT1 under Fe deficiency suggest that BTSL proteins are also active under low Fe conditions. An in-depth biochemical characterization of the $\mathrm{Hr}$ domains is necessary to better understand the possible sensing mechanism of these proteins. Furthermore, the plant proteins have three hemerythrins as opposed to one in mammal FBXL5, suggesting cooperativity between the domains to fine tune the dynamic range of Fe binding affinities. BTSL and BTS may differ in this respect, given the different sequence of the second $\mathrm{Hr}$ domain (Supplementary Figure S2). Only when those questions are addressed will we be able to fully understand the Fe dependent regulation of the E3 ligase activity of BTS/BTSL/HRZ proteins. 


\section{METHODS}

Co-expression analysis

The co-expression networks were produced using publicly available microarray data (NASCArrays; http://affymetrix.arabidopsis.info/) specific from roots and shoots using maccu software (http://maccu.sourceforge.net/). Networks were visualized using Cytoscape 3.0 (http://www.cytoscape.org/). BTSL1, BTSL2 and BTS were used as baits to retrieve coexpressed genes with a Pearson correlation coefficient $>0.60$ for BTSL1 and BTSL2, and $>0.50$ for BTS, and correlations between the retrieved genes were also calculated. Only those genes also present in the published ferrome (Schmidt and Buckhout, 2011) were retained.

\section{Phylogenetic analysis}

Homologous sequences were found by using BLAST, with Arabidopsis BTS, BTSL1, BTSL2 and rice HRZ1 as queries in the Ensembl Plants database (http://plants.ensembl.org). Amino acid alignments were performed using Clustal Omega. A phylogenetic tree was plotted with BioNJ with the Jones-Taylor-Thornton matrix and rendered using TreeDyn 198.3. Species used: Amborella trichocarpa, Arabidopsis thaliana, Brassica oleracea, Hordeum vulgare, Medicago truncatula, Oryza sativa, Physcomitrella patens, Populus trichocarpa, Solanum lycopersicum and Triticum aestivum.

\section{Plant material}

Arabidopsis thaliana ecotype Columbia (Col-0) plants were used as wild type. The T-DNA insertion lines were bts/1-1 (SALK_015054); bts/1-2 (SALK_032464); bts/1-3 (SALK_117926); btsl2-2 (SALK_048470); and bts-1 (SALK_016526); from the Nottingham Arabidopsis Stock Centre. The T-DNA insertion sites were confirmed by sequencing. Double mutant lines were produced by crossing single insertion lines bts/1-1 and bts/1-3 with bts/2-2. Promoter sequences of BTSL1 (-880 nt) and BTSL2 (-2097 nt) were isolated by PCR from genomic DNA using primers BTSL1prom_GWF and BTSL1prom_GWR (BTSL1), and BTSL2pro_GWR and BTSL2pro_GWF (BTSL2). The promoter fragments were cloned upstream of eGFP-GUS in pBGWFS7 (Karimi et al., 2002) and the constructs were stably expressed in wild-type Arabidopsis. pXB2FS7 containing the CaMV 35S promoter upstream of eGFP-GUS was used as a positive control. The bts/1-1 bts/2-2 double mutant line was complemented with tagged versions of BTSL1 and BTSL2. Full coding sequences of BTSL1 and BTSL2 were cloned using the primer pairs BTSL1_B1-F and BTSL1_B1-R, and BTSL2_B1-F and BTSL2_B1-R, respectively. These sequences were cloned upstream eYFP and eGFP, respectively, in the 
vectors pEarleyGate101 and pEarleyGate103 (Earley et al., 2006). All the sequences of the primers used are in Supplementary Table S3.

\section{Plant growth conditions}

For Fe treatments, plants were grown on half Hoagland solution solidified with $0.8 \%(\mathrm{w} / \mathrm{v})$ agar. The medium was composed of (in mM): $\mathrm{K}$ (3.5), $\mathrm{Mg}$ (1), $\mathrm{Ca}(2.5), \mathrm{NO}_{3}(7.5), \mathrm{PO}_{4}(1)$, and $\mathrm{SO}_{4}$ (1); and (in $\mu \mathrm{M}$ ): $\mathrm{Mn}$ (9.14), B (46.3), Mo (0.12), $\mathrm{Zn}(2.4)$ and $\mathrm{Cu}(0.37)$. The pH was adjusted to 5.5 with $1 \mathrm{mM} 2$-(N-morpholino)ethanesulfonic acid (MES). For Fe treatments Fe was added at the desired concentrations using FeEDTA. For Fe deficiency media, no Fe was added and medium was supplemented with $100 \mu \mathrm{M}$ of 3-(2-pyridyl)-5,6-diphenyl-1,2,4-triazine sulfonate (ferrozine). For seed propagation, plants were grown on compost under long-day conditions, $16 \mathrm{~h}$ light at $22^{\circ} \mathrm{C}, 8 \mathrm{~h}$ dark at $20^{\circ} \mathrm{C}$, with $80 \%$ humidity and a light intensity of 140 $-200 \mu \mathrm{mol} \mathrm{m} \mathrm{m}^{-2} \mathrm{~s}^{-1}$.

\section{Promoter GUS studies}

Glucuronidase activity was detected as described (Jefferson et al., 1987), keeping the reaction time constant for equivalent samples. Seedlings were cleared in $100 \%$ ethanol, or fixed with a solution of $3.7 \%(\mathrm{w} / \mathrm{v})$ formaldehyde, $5 \%(\mathrm{v} / \mathrm{v})$ acetic acid, $50 \%(\mathrm{v} / \mathrm{v})$ ethanol, followed by embedding in TechnoVit (Heraeus Kulzer GmbH, Germany), per manufacturer instructions. Transverse root sections $(10 \mu \mathrm{m})$ were imaged on a LEICA DM6000 light microscope. The objectives used were x20/0.7 air or x40/0.85 air and images were collected with a LEICA DFC420C colour camera and processed with LEICA LAS-AF software.

\section{Plant phenotyping}

Arabidopsis leaf pigments were extracted following a modified protocol from Abadía and Abadía (1993). Briefly, leaf pigments were extracted on ice with $80 \%$ acetone, and extracts were filtered and centrifuged to eliminate any particulate. Absorbance was measured at 470 , 645, 662, and $750 \mathrm{~nm}$ wavelengths in a multiplate reader (ClarioStar; BMG Labtech, Germany). Mean values and standard deviations (SD) were obtained from three biological replicates.

Iron content in Arabidopsis leaves was determined by the Ferene method. Dried and powdered samples (4-8 mg) were mineralized at room temperature in $150 \mu \mathrm{L}$ nitric acid (65\%) for 2 days. One hundred and fifty microliters of $\mathrm{H}_{2} \mathrm{O}_{2}(30 \%)$ were added, and the solution was incubated 2 more days. The volume was adjusted to $500 \mu \mathrm{L}$ with sterile water. A standard curve with Fe from 0 to $10 \mu \mathrm{g}$ of Fe from ferric ammonium citrate was subjected to the same mineralization process. One hundred microliters of the mineralized samples were buffered 
with $1 \mathrm{~mL}$ of $15 \%$ ammonium acetate and reduced with $100 \mu \mathrm{L}$ of $4 \%(\mathrm{w} / \mathrm{v})$ ascorbic acid. Then, $\mathrm{Fe}(\mathrm{II})$ was chelated by adding $100 \mu \mathrm{L}$ of $1.5 \% \mathrm{w} / \mathrm{v}$ 3-(2-pyridyl)-5,6-bis(5-sulfo-2-furyl)1,2,4-triazine (Ferene, SIGMA). Absorbance at $593 \mathrm{~nm}$ was measured in triplicates in a multiplate reader (ClarioStar, BMG Labtech).

To localize Fe in tissues, plants always grown on Fe-sufficient media or following a resupply after Fe deficiency induction were incubated with Perls' stain solution (equal volumes of $2 \%$ $[\mathrm{v} / \mathrm{v}] \mathrm{HCl}$ and $2 \%[\mathrm{w} / \mathrm{v}] \mathrm{K}$-ferrocyanide) for $30 \mathrm{~min}$. Seedlings were then washed three times with water, chlorophyll partially removed by incubation with $50 \%$ ethanol and then imaged with a Leica DM6000 microscope.

Fe chelate reductase activity was measured following Yi and Guerinot, (1996). Briefly, roots from 5 plants were blotted dried, weighed and incubated for 30 minutes in the dark with measuring solution containing $0.1 \mathrm{mM} \mathrm{Fe}(\mathrm{III})$-EDTA and $0.3 \mathrm{mM}$ ferrozine in distilled water. $\mathrm{Fe}^{2+}$ production was determined with a spectrophotometer measuring absorbance at $562 \mathrm{~nm}$.

\section{Quantitative reverse-transcription PCR}

Total RNA was extracted using the Plant RNeasy kit (Qiagen), followed by DNase treatment (Turbo DNase kit, Agilent). The integrity of RNA in all samples was verified using agarose gels, and RNA sample purity was analysed by comparing 260/230 nm and 260/280 nm absorbance ratios (Nanodrop 2000, Thermo Fisher). Only samples which passed these quality control checks were used. RNA was quantified using a Qubit 2.0 fluorometer (Thermo Fisher). RNA $(4 \mu \mathrm{g})$ was reverse transcribed to cDNA using Superscript III (Thermo Fisher). RT-qPCR reactions were made using SensiFAST master-mix (Bioline), in $20 \mu$ l volumes, each with 20 ng of cDNA. Reactions were measured in a Bio-Rad CFX-96 real-time PCR system and cycled as per the Bioline protocol. Data were analysed using the Bio-Rad CFX Manager 3.1 software, and were normalized using primer efficiency. All data points are from three or more independent biological replicates, measured in three technical replicates $(n=9)$. The housekeeping genes SAND (AT2G28390) and TIP41-like (AT4G34270) were used as reference genes, as they are unaffected by Fe levels in Arabidopsis (Han et al., 2013).

\section{Protein expression and immunoblot analysis}

Roots from ten plants were ground in liquid nitrogen and suspended in acetone containing $10 \%(\mathrm{w} / \mathrm{v}) \mathrm{TCA}$, mixed thoroughly and precipitated for 2 hours at $-20^{\circ} \mathrm{C}$. Proteins were pelleted and washed three times with cold acetone and then dried prior solubilisation with a buffer containing $8 \mathrm{M}$ urea, $0.5 \%$ SDS, $50 \mathrm{mM}$ DTT and $1 \mathrm{mM}$ PMSF. Proteins were separated on $12.5 \%$ SDS-PAGE gels, blotted into Nitrocellulose membranes and immunodetected using 
anti-Ferritin, anti-FIT and anti-IRT1 (Fan et al., 2014) antibodies and ECL Plus chemiluminescence substrate. Anti-Ferritin antibody was produced using purified pea ferritin for rabbit immunization. For anti-FIT antibody production, the coding sequence of FIT was fused to MBP, purified from $E$. coli and used for rabbit immunization.

Recombinant protein production

For recombinant protein production, full coding sequences of FIT (AT2G28160), bHLH39 (AT3G56980) and bHLH40 (AT4G00120) were cloned from cDNA from Fe deficient Arabidopsis roots or genomic DNA, with a N-terminal His-tag and C-terminal Myc tag. Coding sequences were then inserted into the $\mathrm{pET}-15 \mathrm{~b}$ vector using Ndel and BamHI restriction sites. The C-terminal coding regions of BTSL1 (amino acids 933-1259) and BTSL2 (amino acids 787-1254) were amplified from cDNA from Fe deficient Arabidopsis roots and cloned into the expression vector pMAL-c5X (NEB) using Notl-Sall and Ncol-Sall restriction sites, respectively. This generated an $\mathrm{N}$-terminal fusion with maltose binding protein (MBP) to obtain MBP:BTSL1C and MBP:BTSL2C. A construct for MBP:FIT was produced in the same way. Plasmids were transformed into Escherichia coli Rosetta (NEB). Cells were grown in LB medium and protein expression induced with IPTG (Isopropyl $\beta$-D-1-thiogalactopyranoside) following manufacturer's instructions for $4 \mathrm{~h}$ at $37^{\circ} \mathrm{C}$. The recombinant MBP fusion proteins were purified using amylose resin. All primers used are listed in Supplementary Table S3.

Far-western blot analysis

Soluble protein extracts of bacteria expressing FIT:Myc and bHLH40:Myc proteins were separated in a $10 \%$ SDS PAGE gel and blotted into a nitrocellulose membrane. Membranes were then blocked and incubated with purified MBP, MBP:FIT, MBP:BTSL1C or MBP:BTSL2C at $0.5 \mu \mathrm{M}$ in TBS-tween buffer with $5 \%$ dry milk as blocking agent. After washing, proteins bound to the membrane were detected with anti-Myc (ab18185, Abcam) and ant-MBP (ab23903, Abcam) antibodies, respectively. 


\section{Accession numbers}

BTSL1 (AT1G74770); BTSL2 (AT1G18910); BTS (ATG3G18290);

OsHRZ2 (LOC_Os05g47780); OsHRZ1 (OS01T0689451_02);

TaHRZ1-A (TRIAE_CS42_3AL_TGACv1_195251_AA0646970);

TaHRZ1-B (TRIAE_CS42_3B_TGACv1_220625_AA0712080);

TaHRZ1-D (TRIAE_CS42_3DL_TGACv1_249432_AA0848520);

TaHRZ2-A (TRIAE_CS42_1AL_TGACv1_000799_AA0019360);

TaHRZ2-B (TRIAE_CS42_1BL_TGACv1_031945_AA0123190);

TaHRZ2-D (TRIAE_CS42_1DL_TGACv1_061110_AA0185650).

FIT / bHLH29 (AT2G28160); bHLH38 (AT3G56970); bHLH39 (AT3T56980); bHLH40 (AT4G00120) 


\section{Supplementary data}

Supplementary Table S1. ATG numbers, gene description and correlations between genes appearing the in the networks shown in Supplementary Figure S1.

Supplementary Table S2. ATG numbers, gene description and correlation between genes appearing in the networks shown in Figure 1.

Supplementary Table S3. List of primers.

Supplementary Figure S1. Full co-expression networks of BTSL1, BTSL2 and BTS.

Supplementary Figure S2. Alignment of hemerythrin domains and structure prediction for the second $\mathrm{Hr}$ domain of BTSL1.

Supplementary Figure S3. GUS staining of embryos

Supplementary Fig S4. Fresh weight of WT and bts/ mutant lines under Fe sufficiency and toxicity conditions.

Supplementary Figure S5. Fe reductase activity of bts/1-1, bts/2-2, bts-1 and complementation lines

Supplementary Fig S6. The CHY/RING Zn finger domain of BTSL proteins do not interact with bHLH40. 


\section{AUTHOR CONTRIBUTIONS AND ACKNOWLEDGMENTS}

We are grateful for funding from BBSRC (BB/N001079/1) and the CEPAMS fund. J.R-C. was funded by a Marie Sklodowska Curie fellowship form the EU H2020 programme. We would like to thank Huilan Wu for help with Western blot analysis of FIT and IRT1; Benjamin Planterose for assistance with molecular cloning; the Bioimaging platform and Elaine Barclay for sectioning;

J.R.C. and J.B. conceived the project, performed experiments, analysed the data and wrote the paper. J.M.C., R.T.G. and I.K. performed experiments and analysed data. Y.C. and H.Q.L. contributed new analytical tools. 


\section{REFERENCES}

Abadía, J., and Abadía, A. (1993). Iron and plant pigments. In LL Barton, BC Hemming, eds, Iron Chelation in Plants and Soil Microorganisms, Academic Press, San Diego, pp 327-343.

Barberon, M., Vermeer, J.E.M., De Bellis, D., Wang, P., Naseer, S., Andersen, T.G., Humbel, B.M., Nawrath, C., Takano, J., Salt, D.E., and Geldner, N. (2016). Adaptation of Root Function by Nutrient-Induced Plasticity of Endodermal Differentiation. Cell 164: 447-459.

Barberon, M., Zelazny, E., Robert, S., Conéjéro, G., and Curie, C. (2011). Monoubiquitindependent endocytosis of the IRON-REGULATED TRANSPORTER 1 (IRT1) transporter controls iron uptake in plants. Proc. Natl. Acad. Sci. 108: E450-E458.

Bauer, P., Ling, H.Q., and Guerinot, M. Lou (2007). FIT, the FER-LIKE IRON DEFICIENCY INDUCED TRANSCRIPTION FACTOR in Arabidopsis. Plant Physiol. Biochem. 45: 260-261.

Bournier, M., Tissot, N., Mari, S., Boucherez, J., Lacombe, E., and Briat, J. (2013). Arabidopsis Ferritin 1 (AtFer1) Gene Regulation by the Phosphate Starvation Response 1 (AtPHR1) Transcription Factor Reveals a Direct Molecular Link between Iron and. 288: 22670-22680.

Colangelo, E.P. and Guerinot, M. Lou (2004). The Essential Basic Helix-Loop-Helix Protein FIT1 Is Required for the Iron Deficiency Response. Plant Cell 16: 3400-3412.

Earley, K.W., Haag, J.R., Pontes, O., Opper, K., Juehne, T., Song, K., and Pikaard, C.S. (2006). Gateway-compatible vectors for plant functional genomics and proteomics. Plant J. 45: 616-629.

Fan, H., Zhang, Z., Wang, N., Cui, Y., Sun, H., Liu, Y., Wu, H., Zheng, S., Bao, S., and Ling, H.Q. (2014). SKB1/PRMT5-mediated histone H4R3 dimethylation of lb subgroup bHLH genes negatively regulates iron homeostasis in Arabidopsis thaliana. Plant J. 77: 209-221.

Gayomba, S.R., Zhai, Z., Jung, H., and Vatamaniuk, O.K. (2015). Local and systemic signaling of iron status and its interactions with homeostasis of other essential elements. Front. Plant Sci. 6: 1-13.

Grusak, M.A. and Pezeshgi, S. (1996). Shoot-to-Root Signal Transmission Regulates Root 
Fe(III) Reductase Activity in the dg/ Mutant of Pea. Plant Physiol. 110: 329-334.

Han, B., Yang, Z., Samma, M.K., Wang, R., and Shen, W. (2013). Systematic validation of candidate reference genes for qRT-PCR normalization under iron deficiency in Arabidopsis. BioMetals 26: 403-413.

Hindt, M.N., Akmakjian, Garo Z. Pivarski, K.L., Punshon, T., Baxter, I., Salt, E., Guerinot, M. Lou, and Punshon, T. (2017). BRUTUS and its paralogs, BTS LIKE1 and BTS LIKE2, encode important negative regulators of the iron deficiency response in Arabidopsis thaliana. Metallomics 2.

Iconomou, M. and Saunders, D.N. (2016). Systematic approaches to identify E3 ligase substrates. Biochem. J. 473: 4083-4101.

Jakoby, M., Wang, H.Y., Reidt, W., Weisshaar, B., and Bauer, P. (2004). FRU (BHLH029) is required for induction of iron mobilization genes in Arabidopsis thaliana. FEBS Lett. 577: 528-534.

Jefferson, R.A., Kavanagh, T.A., and Bevan, M.W. (1987). GUS fusions: betaglucuronidase as a sensitive and versatile gene fusion marker in higher plants. EMBO J. 6: 3901-7.

Karimi, M., Inze, D., and Depicker, A. (2002). GATEWAY vectors for Agrobacteriummediated plant transformation. Trends Plant Sci. 7: 193-195.

Kobayashi, T., Nagasaka, S., Senoura, T., Itai, R.N., Nakanishi, H., and Nishizawa, N.K. (2013). Iron-binding haemerythrin RING ubiquitin ligases regulate plant iron responses and accumulation. Nat. Commun. 4: 1-12.

Kobayashi, T. and Nishizawa, N.K. (2015). Intracellular iron sensing by the direct binding of iron to regulators. Front. Plant Sci. 6: 1-4.

Kobayashi, T. and Nishizawa, N.K. (2012). Iron uptake, translocation, and regulation in higher plants. Annual Rev. plant Biol. 63: 131-152.

Lee, H.G. and Seo, P.J. (2016). The Arabidopsis MIEL1 E3 ligase negatively regulates ABA signalling by promoting protein turnover of MYB96. Nat. Commun. 7: 12525.

Leng, R.P., Lin, Y., Ma, W., Wu, H., Lemmers, B., Chung, S., Parant, J.M., Lozano, G., Hakem, R., and Benchimol, S. (2003). Pirh2, a p53-Induced Ubiquitin-Protein Ligase, Promotes p53 Degradation. Cell 112: 779-791. 
Liljegren, S.J., Roeder, A.H.K., Kempin, S.A., Gremski, K., Østergaard, L., Guimil, S., Reyes, D.K., and Yanofsky, M.F. (2004). Control of fruit patterning in Arabidopsis by INDEHISCENT. Cell 116: 843-853.

Lingam, S., Mohrbacher, J., Brumbarova, T., Potuschak, T., Fink-Straube, C., Blondet, E., Genschik, P., and Bauer, P. (2011). Interaction between the bHLH transcription factor FIT and ETHYLENE INSENSITIVE3/ETHYLENE INSENSITIVE3-LIKE1 reveals molecular linkage between the regulation of iron acquisition and ethylene signaling in Arabidopsis. Plant Cell 23: 1815-1829.

Long, T. a, Tsukagoshi, H., Busch, W., Lahner, B., Salt, D.E., and Benfey, P.N. (2010). The bHLH transcription factor POPEYE regulates response to iron deficiency in Arabidopsis roots. Plant Cell 22: 2219-36.

Mai, H., Pateyron, S., and Bauer, P. (2016). Iron homeostasis in Arabidopsis thaliana: transcriptomic analyses reveal novel FIT- regulated genes, iron deficiency marker genes and functional gene networks. BMC Plant Biol. 16: 211-33.

Matthiadis, A. and Long, T.A. (2016). Further insight into BRUTUS domain composition and functionality. Plant Signal. Behav. 11: e1204508.

McElver, J. et al. (2001). Insertional mutagenesis of genes required for seed development in Arabidopsis thaliana. Genetics 159: 1751-1763.

Naseer, S., Lee, Y., Lapierre, C., Franke, R., Nawrath, C., and Geldner, N. (2012). Casparian strip diffusion barrier in Arabidopsis is made of a lignin polymer without suberin. Proc. Natl. Acad. Sci. 109: 10101-10106.

Ravet, K., Touraine, B., Boucherez, J., Briat, J.F., Gaymard, F., and Cellier, F. (2009). Ferritins control interaction between iron homeostasis and oxidative stress in Arabidopsis. Plant J. 57: 400-412.

Rodríguez-Celma, J., Lin, W.-D., Fu, G.-M., Abadía, J., López-Millán, A.-F., and Schmidt, W. (2013a). Mutually exclusive alterations in secondary metabolism are critical for the uptake of insoluble iron compounds by Arabidopsis and Medicago truncatula. Plant Physiol. 162: 1473-85.

Rodríguez-Celma, J., Pan, I.C., Li, W., Lan, P., Buckhout, T.J., and Schmidt, W. (2013b). The transcriptional response of Arabidopsis leaves to Fe deficiency. Front. Plant Sci. 4: $1-10$.

Römheld, V. and Marschner, H. (1986). Evidence for a specific uptake system for iron 
phytosiderophores in roots of grasses. Plant Physiol. 80: 175-180.

Salahudeen, A.A., Thompson, J.W., Ruiz, J.C., Ma, H.-W., Kinch, L.N., Li, Q., Grishin, N. V., and Bruick, R.K. (2009). An E3 Ligase Possessing an Iron-Responsive Hemerythrin Domain Is a Regulator of Iron Homeostasis. Science (80-. ). 326: 722726.

Schikora, A. and Schmidt, W. (2001). Iron Stress-Induced Changes in Root Epidermal Cell Fate Are Regulated Independently from Physiological Responses to Low Iron Availability. Plant Physiol. 125: 1679-1687.

Schmidt, W. and Buckhout, T.J. (2011). A hitchhiker's guide to the Arabidopsis ferrome. Plant Physiol. Biochem. 49: 462-470.

Selote, D., Samira, R., Matthiadis, A., Gillikin, J.W., and Long, T.A. (2015). Iron-binding E3 ligase mediates iron response in plants by targeting basic Helix-Loop-Helix transcription factors. Plant Physiol. 167: 273-286.

Sheng, Y., Laister, R.C., Lemak, A., Wu, B., Tai, E., Duan, S., Lukin, J., Sunnerhagen, M., Srisailam, S., Karra, M., Benchimol, S., and Arrowsmith, C.H. (2008). Molecular basis of Pirh2-mediated p53 ubiquitylation. Nat. Struct. Mol. Biol. 15: 1334-1342.

Shin, L.-J., Lo, J.-C., Chen, G.-H., Callis, J., Fu, H., and Yeh, K.-C. (2013). IRT1 degradation factor1, a ring E3 ubiquitin ligase, regulates the degradation of ironregulated transporter1 in Arabidopsis. Plant Cell 25: 3039-3051.

Sivitz, A., Grinvalds, C., Barberon, M., Curie, C., and Vert, G. (2011). Proteasomemediated turnover of the transcriptional activator FIT is required for plant iron-deficiency responses. Plant J. 66: 1044-1052.

Stenkamp, R.E. (1994). Dioxygen and Hemerythrin. Chem. Rev. 94: 715-726.

Stuart, J.M., Segal, E., Koller, D., and Kim, S.K. (2003). A Gene-Coexpression Network for Global Discovery of Conserved Genetic Modules. Science 302: 249-255.

Tsai, H.-H. and Schmidt, W. (2017). Mobilization of Iron by Plant-Borne Coumarins. Trends Plant Sci. 22: 538-48.

Tzafrir, I., Pena-muralla, R., Dickerman, A., Berg, M., Rogers, R., Hutchens, S., Sweeney, T.C., Mcelver, J., Aux, G., Patton, D., Meinke, D., and Oklahoma, I.T. (2004). Identification of Genes Required for Embryo Development in Arabidopsis. Plant Physiol. 135: 1206-1220. 
Vashisht, A.A. et al. (2009). Control of Iron Homeostasis by an Iron-Regulated Ubiquitin Ligase. Science (80-. ). 326: 718-721.

Vert, G.A., Briat, J.-F., and Curie, C. (2003). Dual Regulation of the Arabidopsis HighAffinity Root Iron Uptake System by Local and Long-Distance Signals. Plant Physiol. 132: $796-804$.

Wang, N., Cui, Y., Liu, Y., Fan, H., Du, J., Huang, Z., Yuan, Y., Wu, H., and Ling, H.Q. (2013). Requirement and functional redundancy of Ib subgroup bHLH proteins for iron deficiency responses and uptake in Arabidopsis thaliana. Mol. Plant 6: 503-513.

Yi, Y. and Guerinot, M. Lou (1996). Genetic evidence that induction of root Fe(III) chelate reductase activity is necessary for iron uptake under iron deficiency. Plant J. 10: 835844.

Yuan, Y., Wu, H., Wang, N., Li, J., Zhao, W., Du, J., Wang, D., and Ling, H.-Q. (2008). FIT interacts with AtbHLH38 and AtbHLH39 in regulating iron uptake gene expression for iron homeostasis in Arabidopsis. Cell Res. 18: 385-97.

Zhang, H.M., Li, Y., Yao, X.N., Liang, G., and Yu, D. (2017). POSITIVE REGULATOR OF IRON HOMEOSTASIS 1 (OsPRI1) positively regulates iron homeostasis in rice. Plant Physiol. 175: 543-54.

Zhang, J., Liu, B., Li, M., Feng, D., Jin, H., Wang, P., Liu, J., Xiong, F., Wang, J., and Wang, H.-B. (2015). The bHLH transcription factor bHLH104 interacts with IAALEUCINE RESISTANT3 and modulates iron homeostasis in Arabidopsis. Plant Cell 27: 787-805. 


\section{FIGURE LEGENDS}

Figure 1. BTSL and BTS are part of different gene expression networks.

(A, B) Co-expression analysis of Fe-responsive genes that are co-regulated with (A) BTSL1 and BTSL2 in roots, and (B) BTS in shoots. The edge thickness relate to the Pearson's correlation coefficient. See Supplemental Table S2 for descriptions of all genes and numerical values.

(C) Expression levels of BTS, BTSL1 and BTSL2 in roots and in shoots under Fe sufficient $(+\mathrm{Fe})$ and $\mathrm{Fe}$ deficient conditions (-Fe). Data were obtained by RNAseq as described in Rodriguez-Celma et al., (2013). RPKM, Reads Per Kilobase Million. The bars represent the mean of $n=3$ biological replicates $\times 10$ seedlings $\pm S D$ ( ${ }^{*} p<0.05$ using a two-tailed $t$-test).

\section{Figure 2. BTSL are uniquely found in dicotyledonous plants.}

(A) Phylogenetic tree of BTS homologs from selected plant species. Sequences were found by BLASTing the amino acid sequences of Arabidopsis BTS, BTSL1, BTSL2 and rice HRZ1 in Ensembl Plants (http://plants.ensembl.org). Species used: Amborella, Amborella trichocarpa; At, Arabidopsis thaliana; Bo, Brassica oleracea; Hv, Hordeum vulgare; Mt, Medicago truncatula; Os, Oryza sativa; Pp, Physcomitrella patens; Pt, Populus trichocarpa; SI, Solanum lycopersicum; Ta, Triticum aestivum. Numbers next to branches indicate bootstrapping values for 100 replications. The scale bar indicates the rate of evolutionary change expressed as number of amino acid substitutions per site.

(B) Domain organization of BTSL, BTS and HRZ proteins.

\section{Figure 3. Promoter activity of BTSL1 and BTSL2 in Fe-deficient roots.}

Promoter sequences of BTSL1 (-880 nt) and BTSL2 (-2097 nt) were inserted upstream of eGFP-GUS and the constructs were stably expressed in wild-type Arabidopsis.

(A) GFP fluorescence in seedlings grown on medium with $50 \mu \mathrm{M}$ FeEDTA (+Fe) or with 100 $\mu \mathrm{M}$ ferrozine to deplete $\mathrm{Fe}(-\mathrm{Fe})$, as indicated, for 6 days. Scale bar is $1 \mathrm{~mm}$.

(B) GUS activity staining in seedlings grown on medium depleted of $\mathrm{Fe}$ (100 $\mu \mathrm{M}$ ferrozine) for 8 days. Scale bar is $0.5 \mathrm{~mm}$ for whole seedlings, $100 \mu \mathrm{m}$ for a close-up of the root tip, and 50 $\mu \mathrm{m}$ for root cross sections.

Images are representative of 3 independent lines for each promoter construct.

Figure 4. BTSL1 and BTSL2 have redundant functions in Fe homeostasis.

(A) Schematic representation of the BTSL1 and BTSL2 genes and mutant alleles. Exons are in black, 5' and 3'-UTRs in grey. T-DNA insertions are indicated by triangles: bts/1-1, SALK_015054; bts/1-2, SALK_032464; bts/1-3, SALK_117926; bts/2-2, SALK_048470. Arrows indicate the left border primer used for genotyping. 
(B, C) Transcript levels of BTSL1 (B) and BTSL2 (C) in wild-type and mutant alleles under standard (+Fe, $50 \mu \mathrm{M}$ FeEDTA) and Fe-deficient conditions (-Fe, $100 \mu \mathrm{M}$ ferrozine). ( ${ }^{*} \mathrm{p}<0.05$ using a two-tailed t-test).

(D) Growth of wild-type and bts/ alleles on medium with varying Fe concentrations. Seeds were germinated on medium with $50 \mu \mathrm{M}$ FeEDTA, and, after 10 days, transferred to medium depleted of Fe using $100 \mu \mathrm{M}$ ferrozine (-Fe) or $50 \mu \mathrm{M}$ FeEDTA; or grown in the presence of 200 or $500 \mu \mathrm{M}$ FeEDTA for 14 days.

(E, F) Chlorophyll concentration (E) under -Fe and control conditions, and iron concentration in shoots (F) from wild type (WT) and bts/ mutant alleles, grown under control and Fe toxicity conditions as in (D). The bars represent the mean of $n=3$ biological replicates $x 10$ seedlings $\pm \mathrm{SD}\left({ }^{*} \mathrm{p}<0.05 ;{ }^{* *} \mathrm{p}<0.01\right.$ using a two-tailed $t$-test $)$.

\section{Figure 5. The bts/ double mutant hyper-accumulates Fe after a period of deficiency.}

(A) Diagram of the Fe treatments used in this study. Seedlings were germinated on agar plates with $50 \mu \mathrm{M}$ FeEDTA. On day 10, seedlings were transferred to a new plate (control treatment) or to medium depleted of $\mathrm{Fe}$ (100 $\mu \mathrm{M}$ ferrozine). After 3 days, seedlings were transferred back to medium with $50 \mu \mathrm{M}$ FeEDTA. Samples were taken at the end of the treatment.

(B) Perls' Prussian Blue staining for Fe in roots in wild-type, bts/1-1 bts/2-2 double mutant and bts-1 seedlings following control (top) and Fe deficiency-resupply treatments (bottom) according to the diagram in (A). The images show a section of the differentiation zone. Scale bar is $50 \mu \mathrm{m}$.

(C) Perls' Prussian Blue staining of leaves after the Fe deficiency-resupply treatment. The images are a close-up of the adaxial leaf surface with veins. Scale bar is $75 \mu \mathrm{m}$.

(D) Immunoblot of ferritin (FER) protein levels in root samples of wild type, bts/1-1 bts/2-2 double mutant and bts-1 seedlings after control and resupply treatments.

(E) Quantification of Fe in the shoots of wild-type, bts/1-1 bts/2-2 double mutant and bts-1 seedlings after control and Fe deficiency-resupply treatments. Bars represent the mean of 3 biological replicates of 10 pooled plants each $\pm S D$ ( ${ }^{*} p<0.05$ using a two-tailed t-test).

\section{Figure 6. The btsI double mutant maintains FRO2 and IRT1 upon Fe resupply.}

(A) Ferric chelate reductase activity of wild-type and bts/1-1 bts/2-2 mutants. Bars represent the mean of 3 biological replicates ( $n=5$ seedlings/assay) $\pm S D\left({ }^{*} p<0.05\right.$ using a two-tailed ttest).

(B) Immunoblot of IRT1 protein levels in root samples in the Fe resupply treatment.

(C, D) Expression of FRO2 (C) and IRT1 (D) in roots from wild-type and bts/1-1 bts/2-2 seedlings determined by quantitative RT-PCR. All values are relative to wild type $+\mathrm{Fe}$. Values are the mean of 3 biological replicates $\pm S E\left({ }^{*} p<0.05\right.$ using a two-tailed t-test). 
Figure 7. FIT protein and $b H L H 38, b H L H 39$ transcript levels are misregulated in the btsI double mutant upon Fe resupply.

(A) Immunoblot of FIT protein levels in root samples of the Fe deficiency-resupply treatment.

(B) Expression of FIT, bHLH38 and bHLH39 in roots from wild-type and bts/1-1 bts/2-2 seedlings determined by quantitative RT-PCR. All values are relative to wild type $+\mathrm{Fe}$. Values are the mean of 3 biological replicates $\pm S E\left({ }^{*} p<0.05\right.$ using a two-tailed t-test).

Figure 8. The CHY/RING Zn finger domain of BTSL proteins interact with FIT.

(A) Diagram of Far-Western blot analysis. Bait proteins with a Myc-tag were produced in $E$. coli and protein extracts separated by SDS-PAGE gel followed by transfer to nitrocellulose membrane. Blots were incubated with purified MBP-tagged proteins of interest, then labelled with anti-MBP antibodies.

(B) Far-Western blots analysis showing direct protein interactions between BTSL proteins and FIT. Bacterial protein extracts with FIT:Myc and bHLH39:Myc were immobilized on nitrocellulose, and the blots were incubated with recombinant MBP, MBP:FIT and the Cterminal domains of BTSL1 and BTSL2, fused to MBP (MBP:BTSL1C and MBP:BTSL2C). FIT is known to form a heterodimer with bHLH39. Ponceau staining shows equal protein loading.

Figure 9. Proposed mode of action of BTSL and BTS in different parts of the plant.

A model of how BTSL and BTS function to control Fe homeostasis. Fe levels are first sensed in the epidermal and cortex cells, where BTSL proteins respond by targeting FIT protein for degradation, thus controlling the uptake of Fe by FRO2 and IRT1 (and other proteins). A second checkpoint to protect plants from excess iron is present in the stele, stem and leaves, where BTS negatively controls ILR3 and bHLH104 protein levels (Selote et al., 2015). How iron affects the activity of BTS/L is not yet known (gray dashed arrows). 


\section{Supplementary data}



B

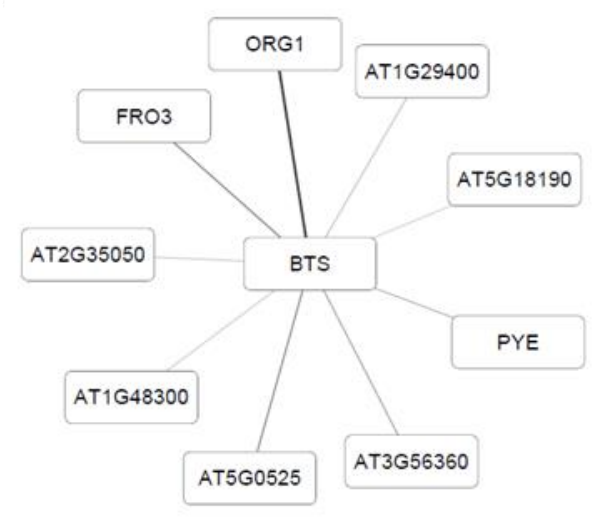

Supplementary Figure $S 1$. Full co-expression networks of BTSL1, BTSL2 and BTS.

(A, B) Co-expression analysis of genes that are co-regulated with (A) BTSL1 and BTSL2 in roots, and (B) BTS in shoots. The edge thickness relate to the Pearson's correlation coefficient. See Supplemental Table $\mathbf{S} 1$ for descriptions of all genes and numerical values. 
bioRxiv preprint doi: https://doi.org/10.1101/231365; this version posted December 8, 2017. The copyright holder for this preprint (which was not certified by peer review) is the author/funder, who has granted bioRxiv a license to display the preprint in perpetuity. It is made available under aCC-BY-NC-ND 4.0 International license.

\section{A}

100

120

160

BTSL1HI2 - . . . . VG RSPIHGLRLF QNAIEKDLRD IQEGLCQAKF QTLILDLDVL - . MARLNFL ADVLVSYSNA FKKFFHPVLE 360 BTSL2 HI2 ….... TG HILVHGIHLW HNAIRKDLVD IQKGLCQLTF PSLSLDLNVL ... VVRLNFL ADVLIFYSNA FKTFFYPVFE 369 BTSL1 Hr3 KNMDDPMIMD VKPIDLLFFF HKAMKMDLDY LVCGSTR - . - . LAADFRFL AEFQQRFHMI KFLYQIHSDA EDEIAFPALE 678 BTSL2 Hr3 KTIGEHLTMD LKPIDLIFYF HKAMKKDLDY LVRGSAR - . - . LATDYSFL GEFQQRFHLI KFLYQIHSDA EDEIAFPALE 673 BTS Hr3 …...... ERPVATIFKF HKAISKDLEF LDVESGKL - . - IDCDGTFI RQFIGRFHLL WGFYKAHSNA EDDILFPALE 725 BTSL1 Hr1 GNTKLSDA- - - PVLFFVYC HKAFRAQLVE LRRFATDAAE AD-SFSGDLA VELSRKFEFL KLVYKYHSAA EDEVIFLALD 99 BTSL2 Hr1 NNARLSDA - - - PILLFVYF HKAFRAQLAE LQFLAGDTVR SG-S - . DLA VELRSKFEFL KLVYKYHSAA EDEVIFSALD 107 BTS Hr1 ANSFSDDAEE ISPILIFLFF HKAVCSELEA LHRLALEFAT GH-HV - DLR L-LRERYRFL RSIYKHHCNA EDEVIFSALD 117 BTS HI2 TNFGSSDT- L LHPVDETKLW HKSTNKEMKE IADEARKIQL SG-DFS-DLS A-FDERLQYI AEVCIFHSLA EDKIIFPAVD 378

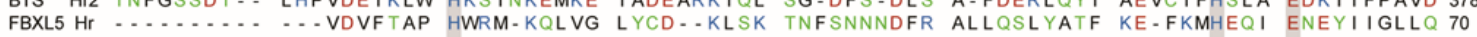
Consensus $-\mathrm{N}$ - ..-D... - - - P-DL - F - F HKAIRKDLVE LQRGA-KL - - - LS-D-D-L AE-Q-RFHFL K-VYK-HSNA EDEIIFPALE

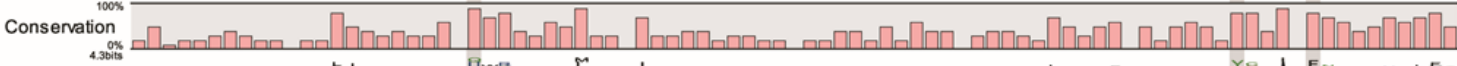

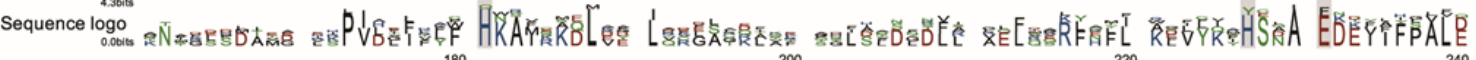
180 200 240

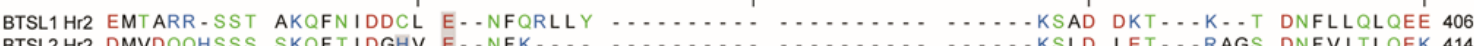


BTSL1 Hr3 - - AKGQLKNI SHSFSIDHEL ETKHFDKVSF ILNEMSELNM L . . . . . . VSTINTTAAD HDR - . KMKY ERLCLSLREI 744 BTSL2 Hr3 - . AKGKLQNI SQSYSIDHEL EVEHLNKVSF LLNELAELNM L . . . . . . V . . . . . LD H H K . - NVKY EKLCMSLQDI 731 BTS Hr3 -.SKETLHNV SHSYTLDHKQ EEKLFGDIYS VLTELSILHE KLQSDSMMED IAQTDTVRTD IDNGDCNKKY NELATKLQGM 803



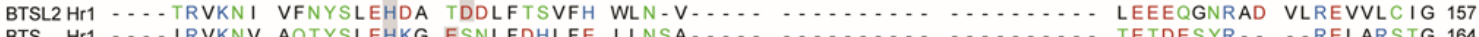



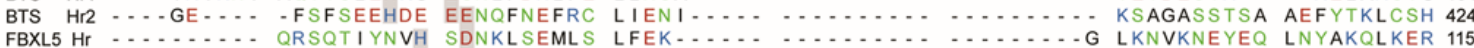

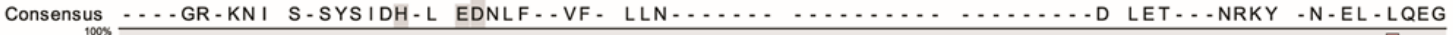
Conservation

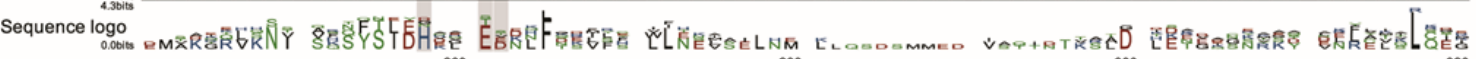
260 280 300

BTSL1Hr2 LESLIIQVTK QFAIQRTEVF PIISKNC-NH EMQKQLLYTS IHVLPLGLLK CVILWFSAHL SEEESQSILH FLSLEDSSPK 485 BTSL2 HI2 LESLILTVAK QFS IEETEVF PIISKNC-NI EMQRQLLYRS IHFLPLGLLK CVIMWFSAOL PEDECOSIIH YLSSEDSFPN 493 BTSL1 Hr3 CKSMHKLLSE HIOHEETELW GLF-RNCFSI EEOEKIIGCM LGRISGEILO DMIPWLMESL TSDEOLAA.. - MSLWROATR 820 BTSL2 Hr3 CKS IHKLLSE HLHREETELW CLF-RDCFT I EEOEKI I ACM LGR ISGE ILO DMIPWLMESL IPDEQHAV.. -MSLWROATR 807 BTS Hr3 CKSIKITLDO HIFLEELELW PLFDKH-FSI BTSL1 Hr1 T . IOSS ICO HMLKEEROVF PLLIEK.FSF REOASLWOF ICSVPVMVLE DFLPWMISHL SHEEKIEVEN CIKDVAP.NE 225 BTSL2 Hr1 T - . IOSSICO HMLKEEROVF PLMIEN - FSF EEOASLWWOF ICSVPVMVLE EIFPWMTSLL SPKEKSEVET CFKEVVP-NE 233 BTS Hr1 A--LOTSVSO HLAKEOKOVF PLLIEK.FKY EEOAYIVWRF LCSIPVNMLA VFLPWISSSI SVDESKEMOT CLKKIVP.GE 240 BTS HI2 ADOIMETIOR HFHNEEIOVL PLARKN - FSF KROQELLYOS LCIMPLRLIE RVLPWLTASL TEDEAKNFLK NLQAGAPKSD 503 FBXL5 Hr LEAFTRDFLP HM-KEEEEVF PMLMEYFTY EELKDIKKKV 1.............. Consensus - - SIQ-..-SQ H-KEETEVF PLFIKN-FSI EEQAK-V-.. ICSIP-E-L - D - - PWL-SSL SEDEQ-EVL- -LSLW-P-TE Conservation

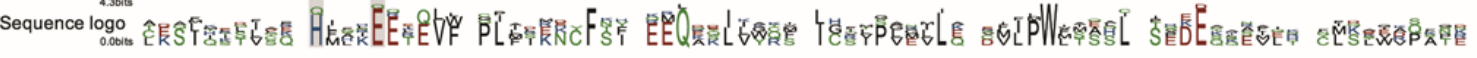

B



Supplementary Figure S2. Alignment of hemerythrin domains and structure prediction for the second $\mathrm{Hr}$ domain of BTSL1.

(A) Alignment of hemerythrin domains of FBXL5, BTS, BTSL1 and BTSL2.

(B) Predicted structure for the second $\mathrm{Hr}$ domain of BTSL1 using Phyre2 server. 

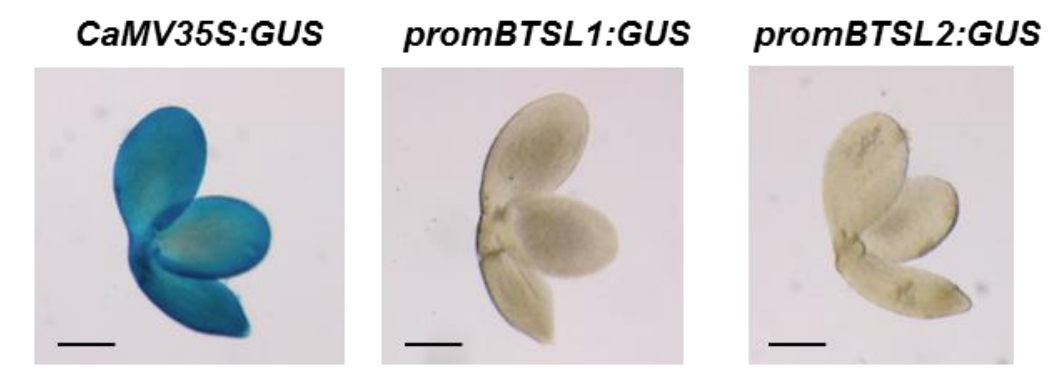

promBTS:GUS

(Selote et al 2015)

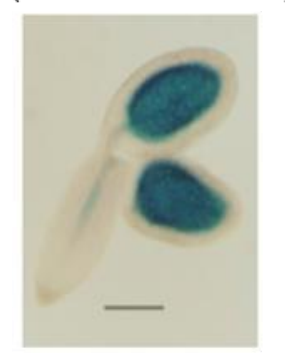

\section{Supplementary Figure S3. GUS staining of embryos}

GUS activity staining of embryos from constitutive CaMV35S:GUS, promBTSL1:GUS and promBTSL2:GUS. Data for promBTS:GUS for comparison from Selote et al., 2015. Scale bar $200 \mu \mathrm{m}$.

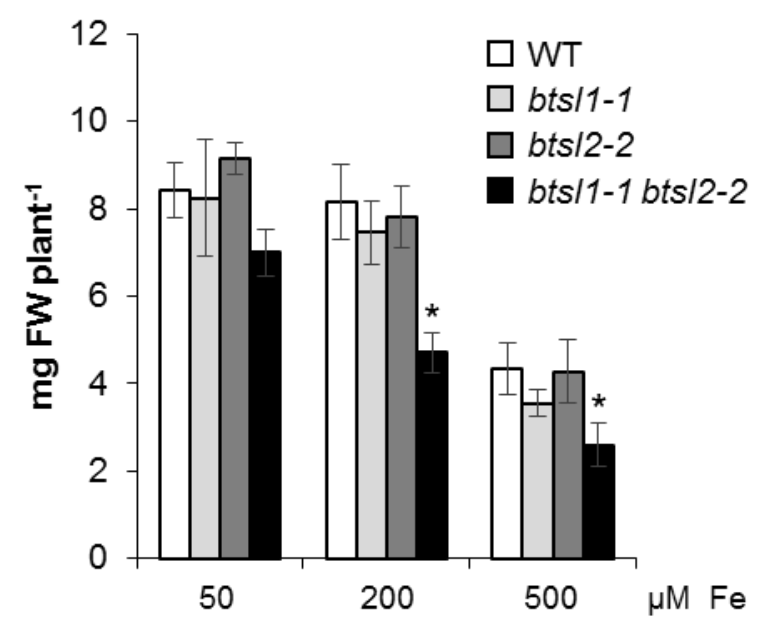

Supplementary Fig S4. Fresh weight of WT and bts/ mutant lines under Fe sufficiency and toxicity conditions

Shoot fresh weight of shoots from wild type (WT) and bts/ mutant alleles, grown under control and Fe toxicity conditions as in Fig 4D. The bars represent the mean of $n=3$ biol. reps $\times 10$ seedlings $\pm S D$. ( ${ }^{*} p<0.05$ using a two-tailed $t$-test). 


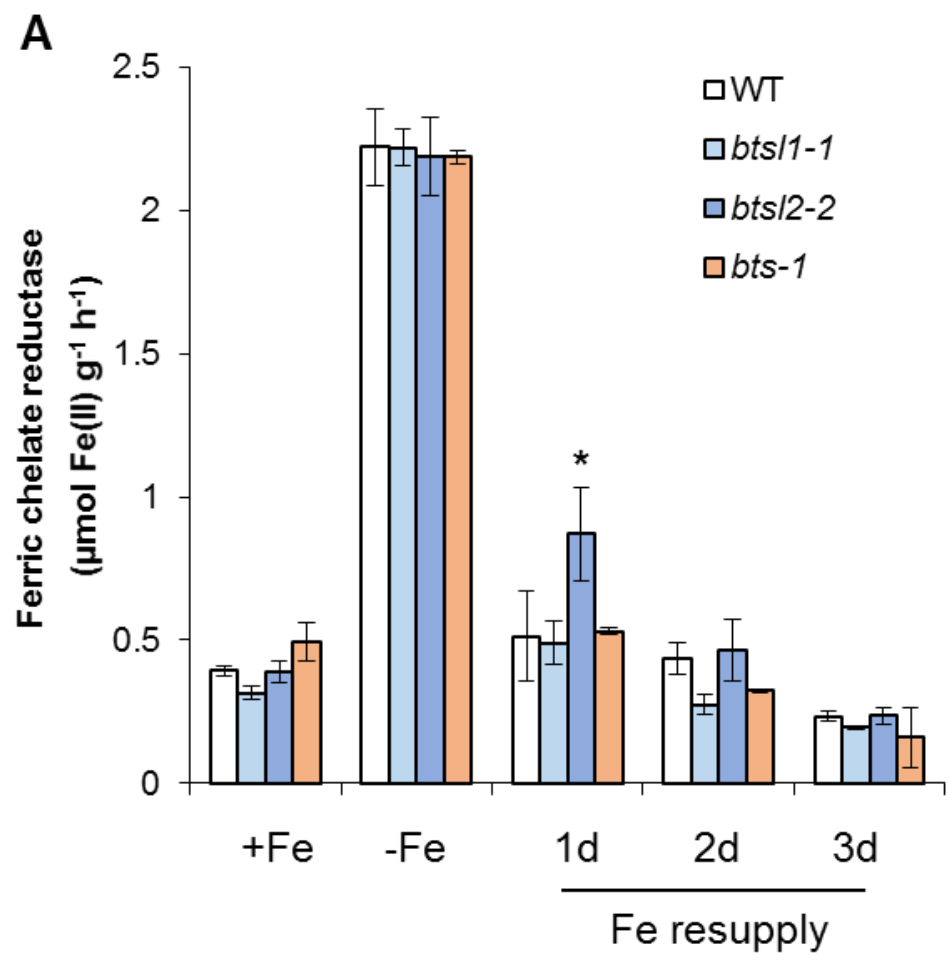

B

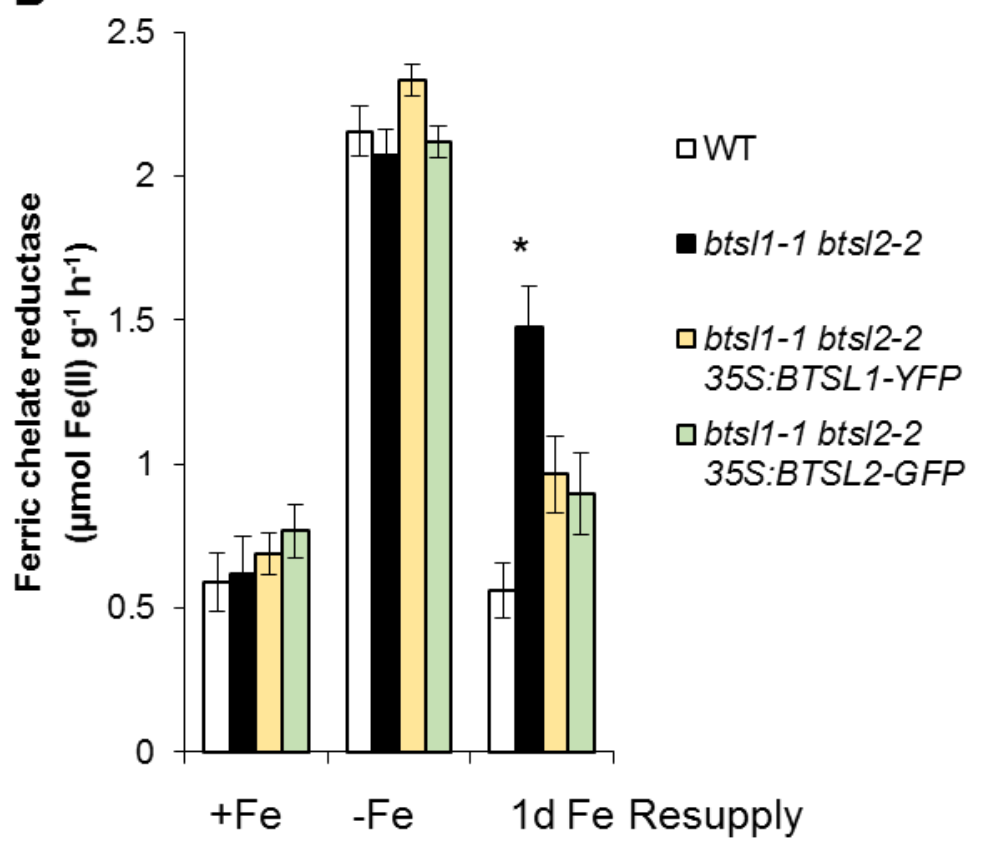

Supplementary Fig S5. Fe reductase activity of bts/1-1, bts/2-2, bts-1 and complementation lines

(A) Ferric chelate reductase activity of bts/1-1, bts/2-2 and bts-1 single insertion mutants under the Fe-defiency and resupply treatment.

(B) Complementation of mutant alleles based on ferric chelate reductase activity. Wild type, bts/1-1 bts/2-2double mutant and bts/1-1 bts/2-2 lines expressing 35S:BTSL1-YFP or 35S:BTSL2-GFP were grown under Fe sufficiency, $\mathrm{Fe}$ deficiency and resupplied with $\mathrm{Fe}$ for 1 day. The results are the mean value of 3 biological replicates $+/$ - SE ( ${ }^{*}, p<0.05$ using a two-tailed Student $t$-test). 


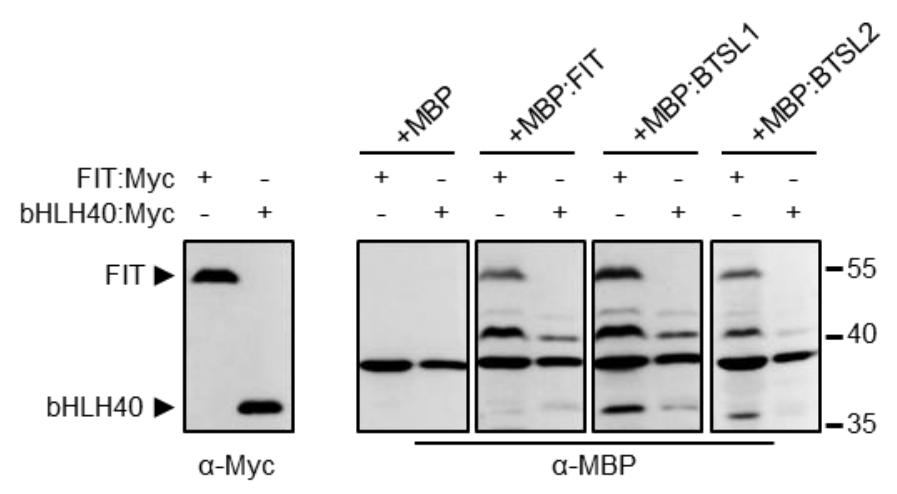

Supplementary Fig S6. The CHY/RING Zn finger domain of BTSL proteins do not interact with bHLH40.

Far-Western blots analysis showing no protein interactions between BTSL proteins and bHLH40. Bacterial protein extracts with FIT:Myc and bHLH40:Myc were immobilized on nitrocellulose, and the blots were incubated with recombinant MBP, MBP:FIT and the Cterminal domains of BTSL1 and BTSL2, fused to MBP (MBP:BTSL1C and MBP:BTSL2C). FIT is known to not form a heterodimer with bHLH40. 
Supplementary Table S1. ATG numbers and gene description of genes appearing in the correlation networks shown in Supplemental Figure S1.

\section{Root co- \\ expression \\ network}

ATG

Symbol Description

Genes co-expressed to BTSL1 and BTSL2

AT3G24290

AT1G07560

AT3G01190

AT5G03570

AT1G47480

AT4G26010

AT4G20460

AT5G55170

AT2G45430

AT3G62040

AT1G51790

AT2G39530

AT3G55150

AT2G21020

AT4G21600

AT1G05250

AT5G48290

AT4G35030

AT3G23190

AT1G05260

AT1G08325

AT2G35270

AT3G13760

AT5G61250

AT1G30900

AT2G21045

AT5G43180

AT3G25930

AT5G40510

AT2G28250

AT1G49310

AT3G13610 protein

IREG2

iron regulated 2

protein 22 protein

(UPF0497)

Unknown

endonuclease 5

Unknown

protein protein

glucuronidase 1

Unknown ammonium transporter $1 ; 5$

Leucine-rich repeat protein kinase family

Peroxidase superfamily protein

alpha/beta-Hydrolases superfamily

Peroxidase superfamily protein

NAD(P)-binding Rossmann-fold

superfamily protein

small ubiquitin-like modifier 3

AT-hook motif nuclear-localized protein

Haloacid dehalogenase-like hydrolase

(HAD) superfamily protein

Leucine-rich repeat protein kinase family

Uncharacterised protein family

exocyst subunit exo70 family protein $\mathrm{H} 1$

Peroxidase superfamily protein

Heavy metal transport/detoxification

superfamily protein

Protein kinase superfamily protein

HR-like lesion-inducing protein-related

Peroxidase superfamily protein

Predicted AT-hook DNA-binding family

Cysteine/Histidine-rich $\mathrm{C} 1$ domain family

VACUOLAR SORTING RECEPTOR 6

Rhodanese/Cell cycle control phosphatase superfamily protein

Protein of unknown function, DUF599

Adenine nucleotide alpha hydrolases-like superfamily protein

Sucrase/ferredoxin-like family protein

Protein kinase superfamily protein

F6H1 2-oxoglutarate (2OG) and Fe(II)dependent oxygenase superfamily protein
Correlation to BTSL1 BTSL2

$0.739 \quad 0.719$

$0.736 \quad 0.826$

$0.734 \quad 0.675$

$0.733 \quad 0.754$

$0.730 \quad 0.749$

$0.728 \quad 0.752$

$0.727 \quad 0.722$

$0.727 \quad 0.828$

$0.726 \quad 0.691$

$0.721 \quad 0.661$

$0.718 \quad 0.646$

$0.718 \quad 0.771$

$0.718 \quad 0.721$

$0.714 \quad 0.694$

$0.712 \quad 0.794$

$0.711 \quad 0.676$

$0.711 \quad 0.688$

$0.711 \quad 0.842$

$0.711 \quad 0.699$

$\begin{array}{ll}0.707 & 0.657\end{array}$

$0.706 \quad 0.670$

$0.703 \quad 0.764$

$0.703 \quad 0.665$

$0.702 \quad 0.676$

$0.702 \quad 0.793$ 
AT2G16750

AT5G17820

AT5G10580

AT3G54040

AT4G15500

AT5G10280

AT1G73270

AT3G58710

AT1G51800

AT5G51060

AT4G20110

AT5G57740

AT5G67400

AT1G13300

AT4G25090

AT3G06300

AT4G15093

AT1G03210

AT5G52830

AT3G13710

AT1G15210

AT2G28970

AT1G61590

AT5G44480

AT4G02270

AT4G16350

AT1G30110

AT1G29280

AT3G60330

AT5G50560

AT5G01610

AT5G25160

AT4G21230

AT5G63600

AT5G40980

AT5G53250

AT1G49390

AT2G42350

AT4G23690

AT1G78090

AT5G26655

AT1G26250
Protein kinase protein with adenine

0.701

0.640

nucleotide alpha hydrolases-like domain

Peroxidase superfamily protein

$0.699 \quad 0.650$

Protein of unknown function, DUF599

PAR1 protein

UDP-Glycosyltransferase superfamily

protein

myb domain protein 92

$0.699 \quad 0.663$

$0.698 \quad 0.733$

$0.697 \quad 0.680$

serine carboxypeptidase-like 6

$0.697 \quad 0.662$

WRKY DNA-binding protein 69

$0.697 \quad 0.747$

Leucine-rich repeat protein kinase family protein

NADPH/respiratory burst oxidase protein

$\mathrm{D}$

VSR7

VACUOLAR SORTING RECEPTOR 7

$0.695 \quad 0.796$

$0.694 \quad 0.671$

$0.693 \quad 0.821$

$0.690 \quad 0.731$

XB3 ortholog 2 in Arabidopsis thaliana

root hair specific 19

$0.689 \quad 0.650$

$0.688 \quad 0.659$

$0.688 \quad 0.726$

protein

Riboflavin synthase-like superfamily

0.686

0.680

protein

$\mathrm{P} 4 \mathrm{H}$ isoform 2

$0.686 \quad 0.805$

catalytic LigB subunit of aromatic ring-

opening dioxygenase family

Phenazine biosynthesis PhzC/PhzF

protein

WRKY DNA-binding protein 27

prenylated RAB acceptor 1.F4

pleiotropic drug resistance 7

0.684

0.738

$0.683 \quad 0.765$

$0.683 \quad 0.737$

$0.683 \quad 0.630$

Leucine-rich repeat protein kinase family

$0.680 \quad 0.823$

$0.678 \quad 0.663$

protein

Protein kinase superfamily protein

$0.678 \quad 0.612$

NAD(P)-binding Rossmann-fold

0.678

0.734

superfamily protein

root hair specific 13

$0.676 \quad 0.628$

calcineurin B-like protein 6

nudix hydrolase homolog 25

WRKY65

WRKY DNA-binding protein 65

$0.676 \quad 0.673$

$0.675 \quad 0.700$

$0.675 \quad 0.785$

$0.675 \quad 0.719$

$0.675 \quad 0.619$

Protein of unknown function (DUF 3339)

Protein of unknown function, DUF538

zinc finger protein 3

cysteine-rich RLK (RECEPTOR-like

protein kinase) 27

FLS5

flavonol synthase 5

$0.674 \quad 0.632$

$0.674 \quad 0.673$

$0.673 \quad 0.696$

$0.672 \quad 0.672$

$0.671 \quad 0.659$

$0.671 \quad 0.688$

arabinogalactan protein 22

0.694

dependent oxygenase superfamily

protein

RING/U-box superfamily protein

$0.669 \quad 0.680$

$0.669 \quad 0.688$

like protein) family protein

trehalose-6-phosphate phosphatase

0.669

0.618

Unknown

0.668

0.633

Proline-rich extensin-like family protein

$0.668 \quad 0.695$ 
AT4G11290

AT5G06800

AT3G27190

AT4G35380

AT1G77330

AT2G34940

AT3G58810

AT1G08670

AT5G06840

AT5G24230

AT1G30870

AT1G74660

AT3G22570

AT1G33800

AT1G67330

AT4G32650

AT4G30670

AT3G23430

AT4G00700

AT1G72300

AT3G15300

AT1G11540

AT3G47040

AT3G05920

AT5G05500

AT5G05790

AT2G29750

AT1G68150

AT5G61340

AT1G76760

AT4G18430

AT5G26310

AT1G02900

AT5G42860

AT5G15180

AT3G61410

AT2G28160 FIT

AT4G11530

AT3G14280

AT1G72120

AT4G18610
Peroxidase superfamily protein

$0.667 \quad 0.695$

myb-like HTH transcriptional regulator

$0.667 \quad 0.645$

family protein

uridine kinase-like 2

$0.667 \quad 0.799$

SEC7-like guanine nucleotide exchange

0.667

0.850 amily protein

2-oxoglutarate (2OG) and Fe(II)-

dependent oxygenase superfamily

protein

VACUOLAR SORTING RECEPTOR 5

$0.666 \quad 0.767$

metal tolerance protein $\mathrm{A} 2$

$0.665 \quad 0.670$

MTPA2

ENTH/VHS family protein

$0.664 \quad 0.643$

Unknown

Lipase class 3-related protein

0.624

$0.663 \quad 0.717$

$0.663 \quad 0.691$

$0.663 \quad 0.623$

$0.663 \quad 0.726$

$0.662 \quad 0.620$

mifunctional inhibitor/lipid-transfer

protein/seed storage $2 \mathrm{~S}$ albumin

superfamily protein

Protein of unknown function (DUF579)

$0.662 \quad 0.761$

Protein of unknown function (DUF579)

$0.662 \quad 0.654$

potassium channel in Arabidopsis

$0.661 \quad 0.766$

thaliana 3

Putative membrane lipoprotein

$0.660 \quad 0.665$

phosphate 1

$0.658 \quad 0.613$

C2 calcium/lipid-binding plant

$0.658 \quad 0.631$

phosphoribosyltransferase family protein

Leucine-rich receptor-like protein kinase family protein

VQ motif-containing protein

$0.657 \quad 0.639$

Sulfite exporter TauE/SafE family protein

Glycosyl hydrolase family protein

Heavy metal transport/detoxification

superfamily protein

Pollen Ole e 1 allergen and extensin

family protein

Duplicated homeodomain-like

superfamily protein

UDP-glucosyl transferase $71 \mathrm{C} 1$

WRKY DNA-binding protein 9

Unknown

thioredoxin $\mathrm{Y} 1$

RAB GTPase homolog A1E

UDP-Glycosyltransferase superfamily

protein

rapid alkalinization factor 1

$0.656 \quad 0.795$

$0.656 \quad 0.689$

$0.656 \quad 0.704$

$0.656 \quad 0.674$

$0.655 \quad 0.628$

0.654

0.657

$0.654 \quad 0.684$

$0.652 \quad 0.815$

$0.652 \quad 0.633$

$0.651 \quad 0.770$

$0.651 \quad 0.755$

$0.650 \quad 0.642$

Unknown

Peroxidase superfamily protein

Unknown

FER-like regulator of iron uptake

cysteine-rich RLK (RECEPTOR-like

protein kinase) 34

Unknown

$0.650 \quad 0.650$

$0.649 \quad 0.683$

$0.649 \quad 0.626$

$0.649 \quad 0.632$

$0.649 \quad 0.748$

$0.647 \quad 0.690$

$0.647 \quad 0.652$

Major facilitator superfamily protein $\quad 0.647 \quad 0.741$

$\begin{array}{lll}\text { Protein of unknown function (DUF640) } & 0.646 & 0.623\end{array}$ 
AT1G09090

AT3G01720

AT4G13235

AT5G67450

AT2G03450

AT5G27920

AT2G32270

AT4G08690

AT1G68940

AT4G17800

AT5G64100

AT3G21710

AT1G01750

AT3G27150

AT3G23200

AT5G25890

AT5G48560

AT1G65610

AT4G22212

AT5G09690

AT1G49860

AT1G51850

AT3G05890

AT3G22850

AT1G12010

AT5G59090

AT4G30170

AT1G47840

AT3G09490

AT1G08990

AT4G10380

AT2G37440

AT5G40730

AT4G20140

AT2G30210

AT2G02960

AT1G14080

AT1G09740

AT5G65210 respiratory burst oxidase homolog $B$

Unknown

$0.646 \quad 0.622$

embryo sac development arrest 21

$0.645 \quad 0.732$

zinc-finger protein 1

$0.644 \quad 0.646$

purple acid phosphatase 9

$0.643 \quad 0.694$

F-box family protein

$0.643 \quad 0.692$

$0.643 \quad 0.733$

ZIP3

zinc transporter 3 precursor

$0.643 \quad 0.665$

Sec14p-like phosphatidylinositol transfer family protein

$0.642 \quad 0.710$

Armadillo/beta-catenin-like repeat family

0.641

0.697

protein

Predicted AT-hook DNA-binding family

0.641

0.745

protein

Peroxidase superfamily protein

$0.641 \quad 0.665$

Unknown

$0.640 \quad 0.711$

actin depolymerizing factor 11

$0.640 \quad 0.691$

Galactose oxidase/kelch repeat

superfamily protein

Uncharacterised protein family

$0.639 \quad 0.758$

(UPF0497)

indole-3-acetic acid inducible 28

0.639

0.709

basic helix-loop-helix (bHLH) DNA-

binding superfamily protein

Six-hairpin glycosidases superfamily

protein

Arabidopsis defensin-like protein

magnesium transporter 7

$0.638 \quad 0.685$

$0.638 \quad 0.801$

0.638

0.727

glutathione S-transferase (class phi) 14

Leucine-rich repeat protein kinase family protein

Low temperature and salt responsive

protein family

Aluminium induced protein with YGL and

LRDR motifs

2-oxoglutarate (2OG) and $\mathrm{Fe}(\mathrm{II})$ -

dependent oxygenase superfamily

protein

subtilase 4.12

$0.637 \quad 0.616$

$0.636 \quad 0.761$

$0.636 \quad 0.640$

$0.635 \quad 0.662$

$0.634 \quad 0.720$

$0.634 \quad 0.791$

$0.633 \quad 0.695$

Peroxidase family protein

hexokinase 3

$0.633 \quad 0.610$

$0.633 \quad 0.663$

$0.631 \quad 0.756$

Tetratricopeptide repeat (TPR)-like

$0.631 \quad 0.711$

superfamily protein

$\begin{array}{lll}\text { plant glycogenin-like starch initiation } & 0.631 & 0.621\end{array}$

protein 5

NOD26-like intrinsic protein 5;1

$0.630 \quad 0.721$

DNAse I-like superfamily protein

arabinogalactan protein 24

$0.629 \quad 0.667$

$0.629 \quad 0.605$

Leucine-rich repeat transmembrane

$0.629 \quad 0.693$

protein kinase

laccase 3

$0.629 \quad 0.612$

RING/FYVE/PHD zinc finger superfamily

0.628

0.694

protein

fucosyltransferase 6

$0.627 \quad 0.769$

Adenine nucleotide alpha hydrolases-like superfamily protein

$0.627 \quad 0.701$

0.627 
AT4G26060

AT5G54140

AT1G70690

AT1G51860

AT3G18560

AT2G39420

AT3G22600

AT3G60070

AT1G70990

AT5G53110

AT3G11550

AT5G20550

AT1G13970

AT3G62780

AT2G34830

AT3G07570

AT2G38390

AT2G21550

AT3G04570

AT1G12160

AT5G25640

AT1G66280

AT5G63990

AT1G30750

AT1G69450

AT1G18970

AT4G14630

AT3G49840

AT2G04430

AT3G19390

AT3G21770

AT3G47210

AT1G66440

AT5G50200

AT3G62280
Ribosomal protein L18ae family

$0.627 \quad 0.721$

IAA-leucine-resistant (ILR1)-like 3

$0.626 \quad 0.740$

Receptor-like protein kinase-related

0.626

0.640

family protein

Leucine-rich repeat protein kinase family $\quad 0.626 \quad 0.684$

protein

Unknown

$0.626 \quad 0.659$

alpha/beta-Hydrolases superfamily

0.625

0.775

protein

Bifunctional inhibitor/lipid-transfer

0.625

0.666

superfamily protein

Major facilitator superfamily protein

proline-rich family protein

$0.625 \quad 0.656$

RING/U-box superfamily protein

Uncharacterised protein family

(UPF0497)

2-oxoglutarate (2OG) and $\mathrm{Fe}(\mathrm{II})$ -

dependent oxygenase superfamily

protein

Protein of unknown function (DUF1336) $\quad 0.622 \quad 0.746$

Calcium-dependent lipid-binding (CaLB $\quad 0.622 \quad 0.631$

domain) family protein

WRKY DNA-binding protein 35

Cytochrome b561/ferric reductase

0.621

0.731

transmembrane with DOMON related

domain

Peroxidase superfamily protein

Bifunctional dihydrofolate

reductase/thymidylate synthase

AT-hook motif nuclear-localized protein

19

Flavin-binding monooxygenase family

protein

Rhomboid-related intramembrane serine

protease family protein

Glycosyl hydrolase superfamily protein

0.621

0.648

$0.621 \quad 0.603$

$0.620 \quad 0.760$

$0.620 \quad 0.746$

$0.620 \quad 0.755$

$0.620 \quad 0.738$

Inositol monophosphatase family protein

Unknown

$0.620 \quad 0.748$

$0.620 \quad 0.809$

$0.619 \quad 0.613$

Early-responsive to dehydration stress

$0.619 \quad 0.716$

protein (ERD4)

germin-like protein 4

$0.619 \quad 0.610$

germin-like protein 9

$0.618 \quad 0.722$

Uncharacterized conserved protein

(DUF2215)

nudix hydrolase homolog 5

$0.618 \quad 0.686$

Granulin repeat cysteine protease family

$0.616 \quad 0.657$

protein

Peroxidase superfamily protein

$0.616 \quad 0.843$

$0.614 \quad 0.631$

$0.613 \quad 0.706$

(DUF247)

Cysteine/Histidine-rich $\mathrm{C} 1$ domain family

$0.613 \quad 0.632$

protein

nitrate transmembrane transporters

$0.613 \quad 0.677$

GDSL-like Lipase/Acylhydrolase

$0.612 \quad 0.719$ 
AT3G20960

AT1G47290

AT2G36890

AT4G15070

AT4G13580

AT2G28670

AT2G27660

AT3G56950

AT2G30840

AT1G63220

AT3G26770

AT1G79530

AT5G45480

AT4G13660

AT1G28680

AT2G41480

AT2G16970

AT5G12880

AT4G15380

AT5G60950

AT4G27270

AT4G13130

AT2G18980 cytochrome P450, family 705 , subfamily

A, polypeptide 33

3beta-hydroxysteroid-

dehydrogenase/decarboxylase isoform 1

Duplicated homeodomain-like

superfamily protein

Cysteine/Histidine-rich $\mathrm{C} 1$ domain family

protein

Disease resistance-responsive (dirigent-

like protein) family protein

Disease resistance-responsive (dirigentlike protein) family protein

Cysteine/Histidine-rich $\mathrm{C} 1$ domain family protein

small and basic intrinsic protein 2;1

2-oxoglutarate (2OG) and $\mathrm{Fe}$ (II)-

dependent oxygenase superfamily

protein

Calcium-dependent lipid-binding (CaLB

domain) family protein

$\mathrm{NAD}(\mathrm{P})$-binding Rossmann-fold

superfamily protein

glyceraldehyde-3-phosphate

dehydrogenase of plastid 1

Protein of unknown function (DUF594)

pinoresinol reductase 2

HXXXD-type acyl-transferase family

protein

Peroxidase superfamily protein

Major facilitator superfamily protein

proline-rich family protein

cytochrome P450, family 705 , subfamily

A, polypeptide 4

COBRA-like protein 5 precursor

Quinone reductase family protein

Cysteine/Histidine-rich $\mathrm{C} 1$ domain family protein

Peroxidase superfamily protein
$0.612 \quad 0.691$

$0.611 \quad 0.666$

$0.610 \quad 0.623$

$0.610 \quad 0.673$

$0.609 \quad 0.602$

$0.609 \quad 0.603$

$0.609 \quad 0.712$

$0.608 \quad 0.683$

$0.607 \quad 0.636$

$0.607 \quad 0.685$

$0.606 \quad 0.722$

$0.606 \quad 0.775$

$0.606 \quad 0.658$

$0.606 \quad 0.642$

$0.605 \quad 0.672$

$0.604 \quad 0.636$

$0.604 \quad 0.607$

$0.603 \quad 0.762$

$0.602 \quad 0.615$

$0.602 \quad 0.772$

$0.601 \quad 0.606$

$0.601 \quad 0.719$

$0.601 \quad 0.605$

Genes co-expressed only with BTSL1

AT5G19970

AT1G72200

AT4G19690 IRT1

AT3G01260

AT4G15390

AT1G62280

AT2G48130

AT4G30450

AT4G00680

AT3G09290
Unknown

0.705

RING/U-box superfamily protein

iron-regulated transporter 1

0.695

0.687

Galactose mutarotase-like superfamily protein

HXXXD-type acyl-transferase family

protein

SLAC1 homologue 1

0.667

0.661

0.656

0.653

protein/seed storage $2 S$ albumin

superfamily protein

glycine-rich protein

0.647

actin depolymerizing factor 8

0.642

telomerase activator 1
0.641 
AT3G61380

AT4G31910

AT1G13830

AT1G48690

AT1G08590

AT2G36100

AT2G27370

AT1G31050

AT5G56540

AT5G24410

AT1G34040

AT4G16400

AT5G27100

AT4G12550

AT4G26320

AT4G29800

AT5G07080

AT3G26610

AT4G22120

AT4G17280

AT5G65970

AT1G12080

AT4G07820

AT1G68710

AT3G45920

AT3G56980

AT1G77690

AT5G25810

AT1G32450

AT2G37180

AT2G29660

AT3G49750

AT5G42500

AT5G56080 NAS2
Phosphatidylinositol N-

0.639

acetyglucosaminlytransferase subunit $\mathrm{P}$ -

related

HXXXD-type acyl-transferase family

0.635

protein

Carbohydrate-binding X8 domain

superfamily protein

Auxin-responsive GH3 family protein $\quad 0.633$

Leucine-rich receptor-like protein kinase $\quad 0.631$

family protein

Uncharacterised protein family

0.631

(UPF0497)

Uncharacterised protein family

(UPF0497)

basic helix-loop-helix (bHLH) DNA-

binding superfamily protein

arabinogalactan protein 14

6-phosphogluconolactonase 4

Pyridoxal phosphate (PLP)-dependent

transferases superfamily protein

Unknown

0.629

0.628

0.627

0.626

0.624

glutamate receptor 2.1

0.622

Auxin-Induced in Root cultures 1

arabinogalactan protein 13

PATATIN-like protein $8 \quad 0.618$

HXXXD-type acyl-transferase family $\quad 0.616$

protein

Pectin lyase-like superfamily protein $\quad 0.615$

ERD (early-responsive to dehydration $\quad 0.614$

stress) family protein

Auxin-responsive family protein $\quad 0.612$

Seven transmembrane MLO family $\quad 0.611$

protein

Vacuolar calcium-binding protein-related $\quad 0.610$

CAP (Cysteine-rich secretory proteins, $\quad 0.608$

Antigen 5, and Pathogenesis-related 1

protein) superfamily protein

ATPase E1-E2 type family protein /

haloacid dehalogenase-like hydrolase

family protein

Protein kinase superfamily protein $\quad 0.608$

bHLH39

basic helix-loop-helix (bHLH) DNA-

binding superfamily protein

0.607

like AUX13

0.607

Integrase-type DNA-binding superfamily $\quad 0.605$

protein

NRT1.5 nitrate transporter 1.5

0.604

Aquaporin-like superfamily protein $\quad 0.603$

zinc finger ( $\mathrm{C} 2 \mathrm{H} 2$ type) family protein $\quad 0.603$

receptor like protein $44 \quad 0.603$

Disease resistance-responsive (dirigent- 0.602

like protein) family protein

nicotianamine synthase 2

0.600 
AT5G05460

AT5G43060

AT4G39270

AT4G26470

AT2G43610

AT2G17720

AT5G15130

AT5G17330

AT1G29330

AT3G51330

AT2G35000

AT2G39200

AT5G58730

AT5G62260

AT4G12080

AT2G44380

AT2G24570

AT5G06750

AT2G41220

AT5G63930

AT2G46140

AT1G74030

AT5G10820

AT3G09940

AT2G31390

AT4G38830

AT2G24610

AT3G60450

AT3G20510

AT2G16595

AT1G50560

AT4G35500

AT2G17290

AT4G21850

AT5G44610

AT1G55730

AT5G04860

AT3G57480

AT2G28890

AT4G28890
Glycosyl hydrolase family 85

Granulin repeat cysteine protease family

0.790

protein

Leucine-rich repeat protein kinase family

0.789

protein

Calcium-binding EF-hand family protein 0.786

Chitinase family protein

0.782

0.780

2-oxoglutarate (2OG) and $\mathrm{Fe}(\mathrm{II})$ -

dependent oxygenase superfamily

protein

WRKY DNA-binding protein 72

0.779

glutamate decarboxylase

0.778

ER lumen protein retaining receptor

0.775

amily protein

Eukaryotic aspartyl protease family

0.769

protein

RING/U-box superfamily protein

0.767

Seven transmembrane MLO family

0.767

protein

pfkB-like carbohydrate kinase family

0.766

protein

AT hook motif DNA-binding family protein

0.765

AT-hook motif nuclear-localized protein 1

0.764

Cysteine/Histidine-rich $\mathrm{C} 1$ domain family

0.762

protein

WRKY DNA-binding protein 17

0.762

0.762

0.761

0.755

Leucine-rich repeat protein kinase family

0.753

Late embryogenesis abundant protein

enolase 1

0.753

0.751

0.746

0.745

pfkB-like carbohydrate kinase family

protein

cysteine-rich RLK (RECEPTOR-like

0.744

protein kinase) 26

cyclic nucleotide-gated channel 14

0.743

Phosphoglycerate mutase family protein

0.742

Transmembrane proteins 14C

0.737

0.735

alpha subunit

cytochrome P450, family 705 , subfamily

0.735

A, polypeptide 25

Protein kinase superfamily protein

0.734

Calcium-dependent protein kinase family

0.734

protein

methionine sulfoxide reductase B9

0.732

0.731

0.731

0.731

0.731

zinc finger (C2H2 type, AN1-like) family

0.730

poltergeist like 4

0.727 
AT1G02530

AT3G24240

AT3G03610

AT2G20780

AT1G71400

AT5G54780

AT5G43370

AT4G39390

AT3G11340

AT1G69810

AT5G58940

AT1G07630

AT3G51440

AT3G01220

AT5G02100

AT4G15400

AT3G54960

AT2G27000

AT3G05580

AT5G10720

AT5G41990

AT2G28960

AT5G39970

AT5G58700

AT5G43520

AT5G14150

AT4G30850

AT2G47140

AT5G18780

AT1G49050

AT2G42060

AT3G53780

AT3G13320

AT2G35120

AT2G46620

AT5G24270

AT3G49860

AT2G44450

AT3G11320
P-glycoprotein 12

0.725

Leucine-rich repeat receptor-like protein

0.724

kinase family protein

ELMO/CED-12 family protein

0.724

Major facilitator superfamily protein

0.723

receptor like protein 12

0.723

0.721

superfamily protein

phosphate transporter 2

0.719

nucleotide sugar transporter-KT 1

0.719

0.718

protein

WRKY DNA-binding protein $36 \quad 0.718$

calmodulin-binding receptor-like $\quad 0.717$

cytoplasmic kinase 1

pol-like 5

0.717

Calcium-dependent phosphotriesterase

0.717

superfamily protein

homeobox protein 20

0.716

Oxysterol-binding family protein

0.715

0.715

protein

PDI-like 1-3

0.714

0.713

A, polypeptide 8

Calcineurin-like metallo-phosphoesterase

0.713

superfamily protein

histidine kinase 5

0.712

0.710

0.710

Leucine-rich repeat protein kinase family

protein

catalytics

0.708

0.707

phospholipase $\mathrm{C} 4$

Cysteine/Histidine-rich C1 domain family $\quad 0.707$

protein

Protein of unknown function, DUF642 0.705

heptahelical transmembrane protein2 0.704

NAD(P)-binding Rossmann-fold 0.704

superfamily protein

F-box/RNI-like superfamily protein $\quad 0.703$

Eukaryotic aspartyl protease family $\quad 0.703$

protein

Cysteine/Histidine-rich C1 domain family $\quad 0.702$

protein

RHOMBOID-like protein $4 \quad 0.701$

cation exchanger $2 \quad 0.701$

Single hybrid motif superfamily protein $\quad 0.701$

P-loop containing nucleoside $\quad 0.700$

triphosphate hydrolases superfamily

protein

Calcium-binding EF-hand family protein $\quad 0.700$

ADP-ribosylation factor-like A1B $\quad 0.700$

beta glucosidase $15 \quad 0.700$

Nucleotide-sugar transporter family $\quad 0.700$

protein 
AT1G02360

AT4G08770

AT2G39410

AT2G20010

AT4G12010

AT1G21370

AT5G59680

AT1G29180

AT5G19520

AT4G32870

AT5G41180

AT5G01040

AT5G12970

AT5G59930

AT3G21510

AT3G53480

AT1G23850

AT1G19360

AT5G24290

AT1G50580

AT5G03630

AT2G14100

AT1G63460

AT1G17500

AT2G44370

AT5G18860

AT5G60280

AT4G01750

AT1G32120

AT2G30340

AT4G18340

AT2G21850

AT3G02880

AT3G52200
Chitinase family protein

0.700

Peroxidase superfamily protein

0.698

alpha/beta-Hydrolases superfamily

0.698

protein

Protein of unknown function (DUF810) 0.698

Disease resistance protein (TIR-NBS- $\quad 0.697$

LRR class) family

Unknown $\quad 0.697$

Leucine-rich repeat protein kinase family $\quad 0.697$

protein

Cysteine/Histidine-rich C1 domain family 0.696

protein

mechanosensitive channel of small

0.696

conductance-like 9

Polyketide cyclase/dehydrase and lipid

0.696

transport superfamily protein

leucine-rich repeat transmembrane

0.696

protein kinase family protein

laccase 8

0.695

Calcium-dependent lipid-binding (CaLB

0.695

family protein

Cysteine/Histidine-rich C1 domain family $\quad 0.694$

protein

histidine-containing phosphotransmitter $1 \quad 0.694$

pleiotropic drug resistance $9 \quad 0.693$

Unknown 0.693

Nucleotide-diphospho-sugar transferase $\quad 0.692$

family protein

Vacuolar iron transporter (VIT) family

0.690

protein

UDP-Glycosyltransferase superfamily

0.689

0.689

0.688

cytochrome P450, family 705 , subfamily

0.688

glutathione peroxidase 8

0.688

ATPase E1-E2 type family protein /

haloacid dehalogenase-like hydrolase

family protein

Cysteine/Histidine-rich $\mathrm{C} 1$ domain family

0.687

protein

inosine-uridine preferring nucleoside

0.686

hydrolase family protein

0.684

family protein

rhamnogalacturonan xylosyltransferase $2 \quad 0.683$

Unknown

0.683

LOB domain-containing protein 13

0.683

Glycosyl hydrolase superfamily protein

0.683

Cysteine/Histidine-rich $\mathrm{C} 1$ domain family

0.681

protein

Leucine-rich repeat protein kinase family

0.681

protein

Dihydrolipoamide acetyltransferase, long

form protein 
AT1G75390

AT3G48410

AT2G16570

AT5G25770

AT5G14000

AT5G38630

AT1G07520

AT1G08350

AT1G71010

AT2G37980

AT1G56145

AT2G02680

AT3G53670

AT4G21830

AT1G54010

AT5G17060

AT1G55380

AT5G51160

AT5G01830

AT2G31030

AT4G01700

AT5G05640

AT1G79450

AT5G13420

AT4G32140

AT1G54890

AT1G17060

AT3G17420

AT4G21410

AT1G67940

AT3G52450

AT3G05990

AT1G47600

AT5G66690

AT3G45650

AT5G57090

AT1G15670

AT5G45510

AT2G45510

AT5G42010

AT3G04060 basic leucine-zipper 44

0.680

alpha/beta-Hydrolases superfamily

0.680

protein

GLN phosphoribosyl pyrophosphate

0.680

amidotransferase 1

alpha/beta-Hydrolases superfamily

0.680

protein

NAC domain containing protein 84

0.680

cytochrome B561-1

0.679

GRAS family transcription factor

0.679

Endomembrane protein 70 protein family

0.678

FORMS APLOID AND BINUCLEATE

0.678

CELLS 1C

O-fucosyltransferase family protein

0.678

Leucine-rich repeat transmembrane

0.678

protein kinase

Cysteine/Histidine-rich $\mathrm{C} 1$ domain family

0.677

protein

Unknown

0.676

methionine sulfoxide reductase B7

0.676

GDSL-like Lipase/Acylhydrolase

0.676

superfamily protein

ADP-ribosylation factor B1B

0.676

Cysteine/Histidine-rich $\mathrm{C} 1$ domain family

0.676

protein

Ankyrin repeat family protein

0.675

0.675

0.674

OSBP(oxysterol binding protein)-related protein 1B

Chitinase family protein

0.674

nucleoprotein-related

0.673

0.673

0.673

Aldolase-type TIM barrel family protein

0.673

EamA-like transporter family

0.673

protein-related

cytochrome p450 72c1

0.673

0.672

0.672

cysteine-rich RLK (RECEPTOR-like

protein kinase) 29

non-intrinsic ABC protein $3 \quad 0.672$

plant U-box 22

0.672

0.672

0.672

0.671

0.671

0.671

0.671

Galactose oxidase/kelch repeat

superfamily protein

Leucine-rich repeat (LRR) family protein

0.671

0.670

A, polypeptide 2

0.670

protein

NAC domain containing protein 46

0.669 
AT1G14780

AT3G21230

AT2G47000

AT1G24180

AT5G54670

AT2G27510

AT3G26470

AT4G33360

AT5G16910

AT1G20900

AT1G62790

AT1G05700

AT2G16760

AT1G30510

AT5G60300

AT2G05260

AT2G17130

AT1G31470

AT5G49680

AT5G11230

AT5G12200

AT5G44790

AT2G44790

AT3G63010

AT1G70170

AT2G24170

AT3G46280

AT3G03520

AT2G35610

AT1G15110

AT5G13110

AT2G34020

AT3G12090

AT1G15380

AT5G39990

AT1G05620
MAC/Perforin domain-containing protein

0.669

4-coumarate:CoA ligase 5

0.669

ATP binding cassette subfamily B4

0.669

Thiamin diphosphate-binding fold (THDP-

0.668

binding) superfamily protein

kinesin 3

0.668

ferredoxin 3

0.668

Powdery mildew resistance protein,

0.668

RPW8 domain

0.668

superfamily protein

cellulose-synthase like D2

0.667

Predicted AT-hook DNA-binding family

0.667

protein

Bifunctional inhibitor/lipid-transfer

0.667

protein/seed storage $2 \mathrm{~S}$ albumin

superfamily protein

Leucine-rich repeat transmembrane

0.667

protein kinase protein

Calcium-dependent phosphotriesterase

0.666

superfamily protein

root FNR 2

0.666

Concanavalin A-like lectin protein kinase

0.666

family protein

alpha/beta-Hydrolases superfamily

0.666

protein

isocitrate dehydrogenase subunit 2

0.665

Major facilitator superfamily protein

0.664

0.663

;RNA pol II promoter Fmp27 protein

domain

Nucleotide-sugar transporter family

0.663

protein

pyrimidine 2

0.662

copper-exporting ATPase / responsive-

0.661

ATPase (RAN1)

uclacyanin 2

0.661

alpha/beta-Hydrolases superfamily

0.661

protein

matrix metalloproteinase

0.660

Endomembrane protein 70 protein family $\quad 0.660$

protein kinase-related

0.660

0.660

0.659

0.659

xyloglucanase 113

phosphatidyl serine synthase family

protein

0.659

0.658

0.657

0.657

Lactoylglutathione lyase / glyoxalase I

0.656

Core-2/l-branching beta-1,6-N-

protein

uridine-ribohydrolase 2 
AT1G64530

AT2G24550

AT5G01620

AT1G24320

AT5G10740

AT3G14470

AT1G30650

AT5G65020

AT5G66280

AT3G09805

AT3G14570

AT2G45330

AT3G25290

AT2G39110

AT5G42630

AT2G23300

AT2G42570

AT1G66450

AT4G31320

AT3G14940

AT5G64370

AT5G65530

AT2G47550

AT5G40760

AT5G07820

AT5G05880

AT2G16530

AT5G59940

AT2G26650

AT1G61360

AT2G26480

AT4G23010

AT4G02180

AT1G29020

AT5G35580

AT1G64060

AT3G54100

AT5G16510

AT4G28650

AT5G02420

AT5G44380

AT5G62150
Plant regulator RWP-RK family protein

0.656

Unknown

0.656

TRICHOME BIREFRINGENCE-LIKE 35

0.655

Six-hairpin glycosidases superfamily

0.654

protein

Protein phosphatase $2 \mathrm{C}$ family protein $\quad 0.654$

NB-ARC domain-containing disease $\quad 0.654$

resistance protein

WRKY DNA-binding protein $14 \quad 0.654$

annexin $2 \quad 0.654$

GDP-D-mannose 4,6-dehydratase $1 \quad 0.653$

Unknown $\quad 0.652$

glucan synthase-like $4 \quad 0.651$

RNA 2'-phosphotransferase, Tpt1 / KptA 0.651

family

Auxin-responsive family protein $\quad 0.651$

Protein kinase superfamily protein $\quad 0.651$

Homeodomain-like superfamily protein $\quad 0.651$

Leucine-rich repeat protein kinase family $\quad 0.651$

protein

TRICHOME BIREFRINGENCE-LIKE $39 \quad 0.651$

Cysteine/Histidine-rich C1 domain family 0.650

protein

SAUR-like auxin-responsive protein $\quad 0.650$

PPC3

family

phosphoenolpyruvate carboxylase $3 \quad 0.649$

beta-ureidopropionase $\quad 0.649$

Protein kinase superfamily protein $\quad 0.649$

Plant invertase/pectin methylesterase $\quad 0.648$

inhibitor superfamily

G6PD6

glucose-6-phosphate dehydrogenase 6

0.648

Plant calmodulin-binding protein-related 0.648

UDP-Glycosyltransferase superfamily $\quad 0.648$

protein

3-oxo-5-alpha-steroid 4-dehydrogenase $\quad 0.648$

family protein

Cysteine/Histidine-rich C1 domain family $\quad 0.648$

protein

$\mathrm{K}+$ transporter 1

0.647

S-locus lectin protein kinase family $\quad 0.647$

protein

UDP-glucosyl transferase 76D1 0.647

UDP-galactose transporter $2 \quad 0.647$

DC1 domain-containing protein $\quad 0.646$

Calcium-binding EF-hand family protein $\quad 0.646$

Protein kinase superfamily protein $\quad 0.646$

respiratory burst oxidase protein $\mathrm{F} \quad 0.645$

O-fucosyltransferase family protein $\quad 0.645$

Alpha-1,4-glucan-protein synthase family $\quad 0.645$

protein

Leucine-rich repeat transmembrane $\quad 0.645$

protein kinase family protein

Unknown $\quad 0.645$

FAD-binding Berberine family protein $\quad 0.645$

peptidoglycan-binding LysM domain- $\quad 0.644$

containing protein 
AT1G30270

AT3G15430

AT3G49210

AT1G34750

AT2G01140

AT5G07390

AT3G44320

AT5G43910

AT2G37280

AT3G26440

AT2G44010

AT1G80360

AT1G54320

AT2G47650

AT1G01640

AT2G14510

AT3G14610

AT2G04100

AT5G35370

AT2G44350

AT1G71697

AT5G16900

AT1G07260

AT5G20400

AT5G58375

AT1G80460

AT5G43390

AT5G15730

AT1G02810

AT2G17370

AT1G01340

AT5G11970

AT5G14690

AT3G03050

AT4G22130

AT5G26595

AT1G14210

AT1G51890

AT3G16340

AT3G47780
CBL-interacting protein kinase 23

0.644

Regulator of chromosome condensation

0.643

(RCC1) family protein

O-acyltransferase (WSD1-like) family

0.643

protein

Protein phosphatase 2C family protein $\quad 0.643$

Aldolase superfamily protein

0.643

0.642

0.642

nitrilase 3

0.642

protein

pleiotropic drug resistance 5

0.642

Protein of unknown function (DUF707) 0.642

Unknown

0.642

0.641

transferases superfamily protein

LEM3 (ligand-effect modulator 3) family

0.641

protein / CDC50 family protein

UDP-xylose synthase 4

0.640

$\mathrm{BTB} / \mathrm{POZ}$ domain-containing protein

0.639

Leucine-rich repeat protein kinase family

0.639

protein

cytochrome P450, family 72 , subfamily A,

0.639

polypeptide 7

MATE efflux family protein

0.639

S-locus lectin protein kinase family

0.639

protein

Citrate synthase family protein

0.638

choline kinase 1

0.638

0.638

protein

UDP-glucosyl transferase $71 \mathrm{C} 3$

0.638

2-oxoglutarate (2OG) and $\mathrm{Fe}(\mathrm{II})$ -

0.638

dependent oxygenase superfamily

protein

Methyltransferase-related protein

0.637

Actin-like ATPase superfamily protein $\quad 0.637$

Uncharacterised conserved protein

0.637

UCP015417, vWA

Protein kinase superfamily protein

0.637

Plant invertase/pectin methylesterase

0.636

inhibitor superfamily

3-hydroxy-3-methylglutaryl-CoA

0.636

reductase 2

cyclic nucleotide gated channel 10

0.635

Protein of unknown function (DUF3511) 0.635

Unknown

0.635

cellulose synthase-like D3

0.635

STRUBBELIG-receptor family $8 \quad 0.635$

Unknown

0.634

Ribonuclease $\mathrm{T} 2$ family protein

0.633

Leucine-rich repeat protein kinase family

0.633

protein

pleiotropic drug resistance 1

0.633

ABC2 homolog 6 
AT3G51460

AT3G15240

AT1G53920

AT4G36990

AT5G11870

AT4G23480

AT1G78000

AT5G01320

AT1G26440

AT1G74210

AT5G60760

AT1G74500

AT1G07000

AT1G02690

AT3G13772

AT2G33630

AT4G37010

AT3G27000

AT3G21240

AT5G40590

AT5G65790

AT3G07690

AT2G18450

AT2G44500

AT3G59660

AT3G50960

AT1G63440

AT5G10520

AT5G58750

AT1G18270

AT3G51350

AT5G63660

AT5G53320

AT5G12340

AT3G51360

AT4G19185
Phosphoinositide phosphatase family

0.633

protein

Serine/threonine-protein kinase WNK

0.633

(With No Lysine)-related

GDSL-motif lipase $5 \quad 0.632$

heat shock factor $4 \quad 0.632$

Alkaline phytoceramidase (aPHC) 0.631

Unknown 0.631

sulfate transporter $1 ; 2 \quad 0.631$

Thiamine pyrophosphate dependent $\quad 0.631$

pyruvate decarboxylase family protein

ureide permease $5 \quad 0.631$

PLC-like phosphodiesterases superfamily $\quad 0.630$

protein

P-loop containing nucleoside

0.630

triphosphate hydrolases superfamily

protein

activation-tagged BRI1(brassinosteroid-

0.630

insensitive 1)-suppressor 1

exocyst subunit exo70 family protein B2 0.630

importin alpha isoform $6 \quad 0.630$

transmembrane nine $7 \quad 0.629$

NAD(P)-binding Rossmann-fold $\quad 0.629$

superfamily protein

centrin $2 \quad 0.629$

actin related protein $2 \quad 0.629$

4-coumarate:CoA ligase $2 \quad 0.628$

Cysteine/Histidine-rich C1 domain family 0.628

protein

myb domain protein 68

6-phosphogluconate dehydrogenase $\quad 0.627$

family protein

succinate dehydrogenase 1-2 0.626

O-fucosyltransferase family protein $\quad 0.626$

C2 domain-containing protein / GRAM 0.626

domain-containing protein

phosducin-like protein 3 homolog $\quad 0.626$

heavy metal atpase $5 \quad 0.626$

ROP binding protein kinases $1 \quad 0.625$

$\mathrm{NAD}(\mathrm{P})$-binding Rossmann-fold $\quad 0.625$

superfamily protein

ketose-bisphosphate aldolase class-II $\quad 0.625$

family protein

Eukaryotic aspartyl protease family $\quad 0.625$

protein

Scorpion toxin-like knottin superfamily $\quad 0.625$

protein

Leucine-rich repeat protein kinase family $\quad 0.624$

protein

Unknown $\quad 0.624$

Eukaryotic aspartyl protease family $\quad 0.623$

protein

nodulin MtN21 /EamA-like transporter 0.622

family protein 
AT1G10050

AT1G25580

AT5G40690

AT2G46700

AT1G76550

AT2G43080

AT2G29330

AT5G03290

AT5G40230

AT1G30720

AT1G78050

AT1G79030

AT1G17430

AT1G48930

AT3G58600

AT5G23720

AT5G01490

AT3G07600

AT3G51160

AT5G62480

AT5G56610

AT3G25585

AT1G67800

AT2G41660

AT1G11580

AT3G49390

AT1G26970

AT3G02360

AT3G57330

AT2G42490

AT5G56630

AT4G02730

AT5G44460

AT5G11920

AT3G04780

AT1G12290

AT5G35200

AT5G41040 glycosyl hydrolase family 10 protein /

0.622

carbohydrate-binding domain-containing

protein

NAC (No Apical Meristem) domain

0.622

transcriptional regulator superfamily

protein

Unknown

0.621

CDPK-related kinase 3

0.621

Phosphofructokinase family protein $\quad 0.621$

$\mathrm{P} 4 \mathrm{H}$ isoform $1 \quad 0.621$

tropinone reductase $\quad 0.621$

isocitrate dehydrogenase $\mathrm{V} \quad 0.621$

nodulin MtN21 /EamA-like transporter $\quad 0.621$

family protein

FAD-binding Berberine family protein $\quad 0.621$

phosphoglycerate/bisphosphoglycerate $\quad 0.620$

mutase

Chaperone DnaJ-domain superfamily $\quad 0.620$

protein

alpha/beta-Hydrolases superfamily

0.620

protein

glycosyl hydrolase $9 \mathrm{C} 1$

0.620

Adaptin ear-binding coat-associated

0.619

protein 1 NECAP-1

dual specificity protein phosphatase

0.619

family protein

CAX4

cation exchanger 4

0.619

Heavy metal transport/detoxification

0.619

superfamily protein

NAD(P)-binding Rossmann-fold

0.619

superfamily protein

GSTU9

glutathione S-transferase tau 9

0.619

Phosphotyrosine protein phosphatases

0.618

superfamily protein

aminoalcoholphosphotransferase

0.618

Copine (Calcium-dependent

0.618

phospholipid-binding protein) family

MIZ1

Protein of unknown function, DUF617

0.618

methylesterase PCR A

0.618

0.618

CTC-interacting domain 10

0.618

Protein kinase superfamily protein

0.617

family protein

autoinhibited Ca2+-ATPase 11

0.617

Copper amine oxidase family protein

0.616

phosphofructokinase 7

0.616

0.616

protein

calmodulin like 43

0.616

0.615

0.615

Protein of unknown function (DUF1000)

0.615

Disease resistance protein (CC-NBS-

LRR class) family

ENTH/ANTH/VHS superfamily protein

0.615

HXXXD-type acyl-transferase family

0.615 
AT1G06840

AT5G08240

AT1G52050

AT1G48510

AT2G34300

AT5G25820

AT3G59100

AT5G19930

AT5G50120

AT5G51590

AT3G05150

AT5G60530

AT5G05440

AT1G27170

AT2G37110

AT1G23140

AT3G07000

AT3G52400

AT1G18980

AT3G50620

AT4G05200

AT5G57480

AT2G26240

AT5G35730

AT5G09890

AT4G08780

AT2G18160

AT5G45490

AT2G28760

AT5G20960

AT5G01450

AT4G10390

AT1G29820

AT5G06740

AT2G30490

AT2G41800

AT5G62165
Leucine-rich repeat protein kinase family

protein

Unknown

Mannose-binding lectin superfamily

protein

Surfeit locus 1 cytochrome c oxidase

biogenesis protein

S-adenosyl-L-methionine-dependent

methyltransferases superfamily protein

Exostosin family protein

0.614

0.614

0.614

0.614

glucan synthase-like 11

0.614

0.614

0.614

transmembrane

0.613

protein

AT hook motif DNA-binding family protein

0.613

0.613

0.612

late embryogenesis abundant protein-

related / LEA protein-related

Polyketide cyclase/dehydrase and lipid

0.612

Pansport superfamily protein

transmembrane receptors;ATP binding

0.612

0.612

0.612

Calcium-dependent lipid-binding (CaLB domain) family protein

Cysteine/Histidine-rich $\mathrm{C} 1$ domain family

0.611

protein

syntaxin of plants 122

0.611

RmIC-like cupins superfamily protein

0.611

P-loop containing nucleoside

0.611

protein

cysteine-rich RLK (RECEPTOR-like

0.611

protein kinase) 25

P-loop containing nucleoside

0.611

triphosphate hydrolases superfamily

protein

Transmembrane proteins $14 \mathrm{C}$

0.611

EXS (ERD1/XPR1/SYG1) family protein

0.611

Protein kinase family protein

0.610

0.610

0.610

basic leucine-zipper 2

0.610

P-loop containing nucleoside

triphosphate hydrolases superfamily

protein

UDP-XYL synthase 6

0.610

aldehyde oxidase 1

0.610

RING/U-box superfamily protein

0.610

0.610

0.609

Magnesium transporter CorA-like family

protein

Concanavalin A-like lectin protein kinase

0.609

family protein

cinnamate-4-hydroxylase

0.609

Protein of unknown function, DUF642

0.609

AGAMOUS-like 42
0.609 
AT5G54650

AT1G50630

AT2G20650

AT1G19230

AT4G28540

AT1G62800

AT2G43040

AT5G04740

AT4G26970

AT4G19030

AT4G25320

AT1G76500

AT5G58690

AT5G38550

AT5G66250

AT4G28370

AT4G29690

AT1G65840

AT4G01480

AT1G18860

AT2G22470

AT1G26640

AT3G02230

AT1G51420

AT3G02140

AT2G19590

AT5G07360

AT3G06420

AT4G30640

AT2G45920

AT3G50300

AT3G23800

AT4G24400

AT1G12000

AT1G79380

AT1G56120

AT3G10640

AT1G53180

AT5G38340

AT5G45500

AT3G52430

AT5G37600

AT1G18390

AT4G35985 formin homology5

0.609

Protein of unknown function (DUF3537)

0.609

RING/U-box superfamily protein

0.609

Riboflavin synthase-like superfamily

0.609

protein

casein kinase I-like 6

0.609

aspartate aminotransferase 4

0.609

tetratricopeptide repeat (TPR)-containing

0.609

protein

ACT domain-containing protein $\quad 0.609$

ACO2

aconitase 2

0.608

NOD26-like major intrinsic protein 1

0.608

0.608

0.607

Predicted AT-hook DNA-binding family

protein

phosphatidylinositol-speciwc

0.607

phospholipase C5

Mannose-binding lectin superfamily $\quad 0.607$

protein

kinectin-related

0.607

RING/U-box superfamily protein

0.606

Alkaline-phosphatase-like family protein

0.606

$\mathrm{PAO} 4$

polyamine oxidase 4

0.606

pyrophosphorylase 5

WRKY DNA-binding protein 61

0.606

0.606

0.605

0.605

0.605

0.604

0.604

AFP2 (ABI five-binding protein 2) family

0.604

ACC oxidase 1

0.604

0.604

0.604

0.603

0.603

HXXXD-type acyl-transferase family

protein

selenium-binding protein 3

0.603

0.603

0.602

0.602

Phosphofructokinase family protein

$\mathrm{Ca}(2)$-dependent phospholipid-binding protein (Copine) family

0.602

protein kinase

SNF7 family protein $\quad 0.602$

Unknown 0.602

Disease resistance protein (TIR-NBS- $\quad 0.602$

LRR class) family

RNI-like superfamily protein

0.602

0.602

protein

GSR 1 glutamine synthase clone R1

0.602

0.602

0.602 
AT4G27860

AT3G04320

AT1G33360

AT4G01910

AT4G30060

AT1G80240

AT5G48940

AT5G48230 vacuolar iron transporter (VIT) family

0.602

protein

Kunitz family trypsin and protease

0.601

inhibitor protein

ATP-dependent Clp protease

0.601

Cysteine/Histidine-rich $\mathrm{C} 1$ domain family

0.601

protein

Core-2/l-branching beta-1,6-N-

0.600

acetylglucosaminyltransferase family

protein

Protein of unknown function, DUF642

0.600

Leucine-rich repeat transmembrane

0.600

protein kinase family protein

acetoacetyl-CoA thiolase 2

0.600

Shoot co-expression network

Correlation to

ATG

Symbol Description

BTS

Genes co-expressed to BTS

AT5G53450 ORG1 OBP3-responsive gene 1

0.733984

AT1G23020 FRO3

ferric reduction oxidase 3

0.644513

AT5G05250

Unknown

0.643582

AT3G56360

Unknown

0.605387

AT3G47640 PYE

basic helix-loop-helix (bHLH) DNA-

0.567715

AT1G29400 protein

0.533767

AT2G35050

MEl2-like protein 5

0.522358

AT1G48300

Protein kinase superfamily protein with

0.509306

AT5G18190

0.501995 
Supplementary Table S2. ATG numbers and gene description of genes appearing in the correlation networks shown in Figure 1.

BTSL1 and BTSL2 root ferrome network

Correlation to

\begin{tabular}{|c|c|c|c|c|c|}
\hline ATG & Symbol & Description & BTSL1 & BTSL2 & BTS \\
\hline AT1G74770 & BTSL1 & BRUTUS-Like 1, zinc ion binding & & & \\
\hline AT1G18910 & BTSL2 & BRUTUS-Like 2, zinc ion binding & & & \\
\hline AT4G19690 & IRT1 & iron-regulated transporter 1 & 0.687 & & \\
\hline AT3G56980 & bHLH39 & $\begin{array}{l}\text { basic helix-loop-helix (bHLH) DNA-binding } \\
\text { superfamily protein }\end{array}$ & 0.607 & & \\
\hline AT3G07720 & Kelch & $\begin{array}{l}\text { Galactose oxidase/kelch repeat superfamily } \\
\text { protein }\end{array}$ & 0.600 & & \\
\hline AT3G53480 & PDR9 & pleiotropic drug resistance 9 & & 0.693 & \\
\hline AT5G35580 & & Unknown & & 0.646 & \\
\hline AT3G21240 & AT4CL2 & 4-coumarate:CoA ligase 2 & & 0.628 & \\
\hline AT5G03570 & IREG2 & iron regulated 2 & 0.765 & 0.711 & \\
\hline AT1G05700 & & $\begin{array}{l}\text { Leucine-rich repeat transmembrane protein } \\
\text { kinase protein }\end{array}$ & 0.742 & 0.667 & \\
\hline AT3G13610 & $\mathrm{F} 6 \mathrm{H} 1$ & $\begin{array}{l}\text { 2-oxoglutarate }(2 \mathrm{OG}) \text { and } \mathrm{Fe}(\mathrm{II}) \text {-dependent } \\
\text { oxygenase superfamily protein }\end{array}$ & 0.702 & 0.793 & \\
\hline AT3G60330 & $\mathrm{HA} 7$ & $\mathrm{H}(+)$-ATPase 7 & 0.675 & 0.719 & \\
\hline AT3G58810 & MTPA2 & metal tolerance protein $\mathrm{A} 2$ & 0.664 & 0.643 & \\
\hline AT2G29750 & & Unknown & 0.654 & 0.684 & \\
\hline AT3G61410 & & Unknown & 0.649 & 0.632 & \\
\hline AT2G28160 & FIT & FER-like regulator of iron uptake & 0.649 & 0.748 & \\
\hline AT2G32270 & ZIP3 & zinc transporter 3 precursor & 0.643 & 0.665 & \\
\hline AT3G18560 & & Unknown & 0.626 & 0.659 & \\
\hline \multicolumn{3}{|c|}{ BTS shoot ferrome network } & \multicolumn{3}{|c|}{ Correlation to } \\
\hline ATG & Symbol & Description & BTSL1 & BTSL2 & BTS \\
\hline AT3G18290 & BTS & BRUTUS, zinc finger protein-related & & & \\
\hline AT5G53450 & ORG1 & OBP3-responsive gene 1 & & & 0.734 \\
\hline AT1G23020 & FRO3 & ferric reduction oxidase 3 & & & 0.645 \\
\hline AT5G05250 & & Unknown & & & 0.644 \\
\hline AT3G47640 & PYE & $\begin{array}{l}\text { basic helix-loop-helix (bHLH) DNA-binding } \\
\text { superfamily protein }\end{array}$ & & & 0.568 \\
\hline
\end{tabular}


Supplementary Table S3. List of primers.

\begin{tabular}{|c|c|c|c|}
\hline $\begin{array}{l}R T-P C R \\
\text { ATG }\end{array}$ & Gene & Primer name & Sequence \\
\hline AT4G34270 & TIP41-like & AtTIP41F & GTGAAAACTGTTGGAGAGAAGCAA 1 \\
\hline AT4G34270 & TIP41-like & AtTIP41R & TCAACTGGATACCCTTTCGCA 1 \\
\hline AT2G28390 & SAND & AtSANDF & AACTCTATGCAGCATTTGATCCACT 1 \\
\hline AT2G28390 & SAND & AtSANDR & TGATTGCATATCTTTATCGCCATC ${ }^{1}$ \\
\hline AT1G01580 & AtFRO2 & qS-FRO2F & TTACCCGATCGACCACAACAC \\
\hline AT1G01580 & AtFRO2 & qA-FRO2R & CCGCACTACAAGTCGCCATTAT \\
\hline AT4G19690 & AtIRT1 & qS-IRT1F & CACCATTCGGAATAGCGTTAGG \\
\hline AT4G19690 & AtIRT1 & qA-IRT1R & CCAGCGGAGCATGCATTTA \\
\hline AT5G01600 & AtFER1 & AtFER1F & TCGTTGAGAGTGAATTTCTGG ${ }^{2}$ \\
\hline AT5G01600 & AtFER1 & AtFER1R & ACCCCAACATTGGTCATCTG ${ }^{2}$ \\
\hline AT1G74770 & AtBTSL1 & BTSL1Fnew & GGCAATGAAGATGGATTTGG \\
\hline AT1G74770 & AtBTSL1 & BTSL1Rnew & TCATATGGAACCGTTGCTGA \\
\hline AT1G18910 & AtBTSL2 & BTSL2Fnew & CGGGGCAGAATCCATCTTAT \\
\hline AT1G18910 & AtBTSL2 & BTSL2Rnew & GTTGCAACAAGGAGCAAGAAG \\
\hline AT2G28160 & FIT & FIT1setAF & TCGCGGGTCTTGAAGCTTCT \\
\hline AT2G28160 & FIT & FIT1setAR & GCAGGAGGATTGATACCGCG \\
\hline AT3G56970 & bHLH38 & bHLH38_QA & CTGACGAAACAGATACTCCCAAGCT \\
\hline AT3G56970 & bHLH38 & bHLH38_QS & TTTCACAAACTTCGGTTGGCC \\
\hline $\begin{array}{l}\text { Genotyping } \\
\text { ATG }\end{array}$ & line & Primer name & Sequence \\
\hline AT1G74770 & bts/1-1 & btsl1-1_LP & TGCTTGGCATAATCCTTCTTG \\
\hline AT1G74770 & $b t s / 1-1$ & btsl1-1_RP & GAACTCTCTTGCTTCCTGAAGC \\
\hline AT1G74770 & $b t s / 1-1$ & btsl1-2_LP & GACTATCGTCAAGCAGCCAAG \\
\hline AT1G74770 & $b t s / 1-1$ & btsl1-2_RP & GATGCTCCGGTTCTTTTCTTC \\
\hline AT1G74770 & $b t s / 1-1$ & btsl1-3_LP & GGCTGACTCAGCTAATCTCCC \\
\hline AT1G74770 & $b t s / 1-1$ & btsl1-3_RP & TGCAACACGAATTTAGACAAGG \\
\hline AT1G18910 & btsl2 & bts2_LP & TCGGTTATTCAGGCAAAACAC \\
\hline AT1G18910 & bts/2 & bts2_RP & CCCTTTGTACTCATCAGCAGC \\
\hline AT3G18290 & $b t s-1$ & bts-1_LP & CCAAATGCGTTCGTAGGTAAG \\
\hline AT3G18290 & bts-1 & bts-1_RP & TCAGATTTACACAAATTTGCAGC \\
\hline
\end{tabular}

\begin{tabular}{|c|c|c|c|}
\hline \multicolumn{4}{|c|}{ Promoter-GUS } \\
\hline ATG & Promoter & Primer name & Sequence \\
\hline AT1G74770 & BTSL1 & $\begin{array}{l}\text { BTSL1prom_G } \\
\text { WF }\end{array}$ & $\begin{array}{l}\text { GGGGACAAGTTTGTACAAAAAAGCAGGC } \\
\text { TAGTATTGATTTTGTGAGCCCAATTAAC }\end{array}$ \\
\hline AT1G74770 & BTSL1 & $\begin{array}{l}\text { BTSL1prom_G } \\
\text { WR }\end{array}$ & $\begin{array}{l}\text { GGGGACCACTTTGTACAAGAAAGCTGGG } \\
\text { TTCCACCGCAACAACAATAACGGTG }\end{array}$ \\
\hline AT1G18910 & BTSL2 & BTSL2pro_GWF & $\begin{array}{l}\text { GGGGACAAGTTTGTACAAAAAAGCAGGC } \\
\text { TAAATTCATATAAGAATTGCTTTATAC }\end{array}$ \\
\hline AT1G18910 & BTSL2 & $\begin{array}{l}\text { BTSL2pro_GW } \\
\text { R }\end{array}$ & $\begin{array}{l}\text { GGGGACCACTTTGTACAAGAAAGCTGGG } \\
\text { TCCACCATGAACAGGAGAGGAGGTG }\end{array}$ \\
\hline $\begin{array}{l}\text { BTSL-GFP } \\
\text { ATG }\end{array}$ & Gene & Primer name & Sequence \\
\hline AT1G74770 & BTSL1 & BTSL1_B1-F & $\begin{array}{l}\text { GGGGACAAGTTTGTACAAAAAAGCAGGC } \\
\text { TTGATGGGAGGCGGAAA }\end{array}$ \\
\hline AT1G74770 & BTSL1 & BTSL1_B1-R & $\begin{array}{l}\text { GGGGACCACTTTGTACAAGAAAGCTGGG } \\
\text { TTAAGGAGCCTTGAGTT }\end{array}$ \\
\hline
\end{tabular}

\footnotetext{
1 Han, B., Yang, Z., Samma, M.K., Wang, R., and Shen, W. (2013). Systematic validation of candidate reference genes for qRT-PCR normalization under iron deficiency in Arabidopsis. BioMetals 26: 403-413.

2 Bournier, M., Tissot, N., Mari, S., Boucherez, J., Lacombe, E., and Briat, J. (2013). Arabidopsis Ferritin 1 (AtFer1) Gene Regulation by the Phosphate Starvation Response 1 (AtPHR1) Transcription Factor Reveals a Direct Molecular Link between Iron and. 288: 22670-22680.
} 


$\begin{array}{llll}\text { AT1G18910 } & \text { BTSL2 } & \text { BTSL2_B1-F } & \begin{array}{l}\text { GGGGACAAGTTTGTACAAAAAAGCAGGC } \\ \text { TTGATGGGAGTCGGAGA }\end{array} \\ \text { AT1G18910 } & \text { BTSL2 } & \text { BTSL2_B1-R } & \begin{array}{l}\text { GGGGACCACTTTGTACAAGAAAGCTGGG } \\ \text { TTGAAAAGTCTGGTGTT }\end{array}\end{array}$

Recombinant protein production

\begin{tabular}{|c|c|c|c|}
\hline ATG & Gene & Primer name & Sequence \\
\hline AT2G28160 & FIT & FITmyc_F & ACTCATATGGAAGGAAGAGTCAACGCTC \\
\hline AT2G28160 & FIT & FITmyc_R & $\begin{array}{l}\text { CGCGGATCCCTACAGATCTTCTTCAGAAAT, } \\
\text { AGTTTTTGTTCAGTAAATGACTTGATGAATT } \\
\text { AAAACC }\end{array}$ \\
\hline AT4G00120 & bHLH40 & 40myc_F & $\begin{array}{l}\text { ACTCATATGGAAAATGGTATGTATAAAAAGF } \\
\text { AAGG }\end{array}$ \\
\hline AT4G00120 & bHLH4O & 40myc_R & $\begin{array}{l}\text { CGCGGATCCCTACAGATCTTCTTCAGAAAT, } \\
\text { AGTTTTTGTTCGGGTTGGGAGTTGTGGTAA } \\
\text { AAC }\end{array}$ \\
\hline $\begin{array}{l}\text { AT1G74770 } \\
\text { AT1G74770 }\end{array}$ & $\begin{array}{l}\text { BTSL1 } \\
\text { BTSL1 }\end{array}$ & $\begin{array}{l}\text { MBP-1CT_F } \\
\text { MBP-1CT_R }\end{array}$ & $\begin{array}{l}\text { ACTCCATGGCTCAGAAGGTTAGCCAGTTTG } \\
\text { TGAGTCGACTTATTTTTCGAACTGCGGGTG } \\
\text { GCTCCAAAGGAGCCTTGAGTTGTAGGAG }\end{array}$ \\
\hline $\begin{array}{l}\text { AT1G18910 } \\
\text { AT1G18910 }\end{array}$ & $\begin{array}{l}\text { BTSL2 } \\
\text { BTSL2 }\end{array}$ & $\begin{array}{l}\text { MBP-2CT_F } \\
\text { MBP-2CT_R }\end{array}$ & $\begin{array}{l}\text { ACTCCATGGAATCTTTGATCCCTGATGAAC } \\
\text { TGAGTCGACTTATTTTTCGAACTGCGGGTG } \\
\text { GCTCCAGAAAAGTCTGGTGTTGTAGGAGGC }\end{array}$ \\
\hline
\end{tabular}

